\title{
UNIFORM INTERIOR ERROR ESTIMATES FOR THE REISSNER-MINDLIN PLATE MODEL
}

\author{
LUCIA GASTALDI
}

\begin{abstract}
Interior error estimates are derived for the solution of the ReissnerMindlin plate model discretized by mixed-interpolated elements. Precisely, it is shown that the error in an interior domain can be estimated by the sum of two terms: the first has the best order of accuracy that is possible locally for the finite element spaces used, the second is a weak norm of the error on a slightly larger domain (this term measures the effects from outside of this domain). The analysis is based on some abstract properties enjoyed by the finite element spaces considered.
\end{abstract}

\section{INTRODUCTION}

The Reissner-Mindlin model describes the deformation of a clamped plate of moderate thickness subject to a transverse loading. The plate occupies, in the undeformed configuration, a region $\Omega \times(-t, t)$, where $\Omega$ is a regular, bounded domain in $\mathscr{R}^{2}$ and $t>0$ is the thickness of the plate. Given a vertical load $F$, the problem consists in determining the rotation $\theta$ of the fibers normal to $\Omega$ and the transverse displacement $w$, as the minimizers in $\left[\mathbf{H}_{0}^{1}(\Omega)\right]^{2} \times \mathbf{H}_{0}^{1}(\Omega)$ of the following functional:

$$
\mathbf{J}_{t}(\boldsymbol{\theta}, w)=\frac{t^{3}}{2} a(\boldsymbol{\theta}, \boldsymbol{\theta})+\frac{\lambda t}{2}\|\boldsymbol{\theta}-\operatorname{grad} w\|_{0}^{2}-t^{3}(F, w),
$$

where $\left(t^{3} / 2\right) a(\boldsymbol{\theta}, \boldsymbol{\theta})$ is the bending energy, $\frac{\lambda t}{2}\|\boldsymbol{\theta}-\operatorname{grad} w\|_{0}^{2}$ is the shear energy, and $\|\cdot\|_{0}$ and $(\cdot, \cdot)$ represent respectively the norm and the inner product in $\mathbf{L}^{2}(\Omega)$. Here, $\lambda=\frac{E k}{2(1+\nu)}$, with $E$ the Young modulus, $\nu$ the Poisson ratio, and $k$ the shear correction factor. Moreover, note that the load has been scaled so that the solution tends to a nonzero limit as $t$ tends to zero. See for instance [3] for more details.

We consider the finite element approximation of the Reissner-Mindlin plate proposed recently in [6]. The discretized problem is described by: find $\boldsymbol{\theta}_{h} \in \boldsymbol{\Theta}_{h}$ and $w_{h} \in W_{h}$, which minimize the following functional:

$$
\mathbf{J}_{t h}\left(\boldsymbol{\theta}_{h}, w_{h}\right)=\frac{t^{3}}{2} a\left(\boldsymbol{\theta}_{h}, \boldsymbol{\theta}_{h}\right)+\frac{\lambda t}{2}\left\|\mathbf{R} \boldsymbol{\theta}_{h}-\operatorname{grad} w_{h}\right\|_{0}^{2}-t^{3}\left(F, w_{h}\right) .
$$

Received by the editor December 13, 1991 and, in revised form, May 19, 1992.

1991 Mathematics Subject Classification. Primary 65N30, 73K10, 73V05.

Partially supported by IAN-CNR (Pavia) and MURST 40\% Analisi Numerica e Matematica Computazionale (Italy). 
Here, $\boldsymbol{\Theta}_{h}$ and $W_{h}$ are finite element subspaces of $\left[\mathbf{H}_{0}^{1}(\Omega)\right]^{2}$ and $\mathbf{H}_{0}^{1}(\Omega)$, respectively, and $\mathbf{R}$ is a linear bounded operator which takes values in a third finite element space $\boldsymbol{\Gamma}_{h}$ and satisfies $\mathbf{R} \operatorname{grad} w_{h}=\operatorname{grad} w_{h} \forall w_{h} \in W_{h}$. Notice that in the shear energy we used $\left\|\mathbf{R} \boldsymbol{\theta}_{h}-\operatorname{grad} w_{h}\right\|_{0}^{2}$ instead of $\left\|\boldsymbol{\theta}_{h}-\operatorname{grad} w_{h}\right\|_{0}^{2}$ in order to avoid the numerical locking phenomenon.

This finite element discretization is characterized by the choice of the finite element spaces $\boldsymbol{\theta}_{h}, W_{h}, \Gamma_{h}$, and by the choice of the linear operator $\mathbf{R}$. In [6] a whole family is constructed combining known results on the approximation of Stokes problems with known results on the approximation of linear elliptic problems. This idea points to some properties which are such that if an approximation satisfies them, then the discrete solution converges to the continuous solution uniformly in $t$. Moreover, in [8] and [9], the following optimal error estimates were proved:

$$
\left\|\boldsymbol{\theta}-\boldsymbol{\theta}_{h}\right\|_{1}+\left\|w-w_{h}\right\|_{1} \leq c h^{s}\left(\|\boldsymbol{\theta}\|_{s+1}+\|w\|_{s+1}\right),
$$

with $C$ a suitable constant, independent of $h$ and $t$.

Unfortunately, these estimates are not uniform with respect to $t$, unless $s \leq 3 / 2$. In fact, Arnold and Falk have investigated in [1] and [2] the structure of the solution of the Reissner-Mindlin plate equations in its dependence on the plate thickness, showing the existence of a boundary layer for $t$ small and a uniform bound for $\|\boldsymbol{\theta}\|_{5 / 2}$ but not for more regular spaces.

This leads us to think that it is not useful to introduce finite element spaces with improved properties of approximation, because such boundary layers limit their accuracy anyhow. But, numerical experiments have shown good accuracy away from the boundary and, on the other hand, the boundary layer does not limit the regularity of the solution at a positive distance from $\partial \Omega$ nor does it affect the smoothness of its restriction to $\partial \Omega$.

Therefore, our aim is to obtain interior estimates which are uniform with respect to $t$, so that they can take advantage of the richness of the finite element spaces.

Using the technique suggested by Nitsche and Schatz (see [14]) of localizing the problem by means of some cutoff functions, we prove that the error in a compact subset of $\Omega$ can be estimated by the sum of two terms: the first has the best order of accuracy that is possible locally for the subspaces used, the second is a weak norm of the error on a slightly larger domain which measures the effects from outside of the compact subset in consideration. The analysis will be carried out using essentially the abstract properties of the finite element spaces, combined with some local approximability assumptions concerning the subspaces containing the functions with compact support in $\Omega$.

\section{Notations}

We list here the basic notations used in the paper.

Let $A$ be a bounded open set in $\mathscr{R}^{2}$. For $s \geq 0$ any real number, $\mathbf{H}^{s}(A)$ and $\mathbf{H}_{0}^{s}(A)$ will denote the usual $\mathbf{L}^{2}$-based Sobolev spaces $\left(\mathbf{H}^{0}(A)=\mathbf{L}^{2}(A)\right)$ and \|\|$_{s, A}$ will stand for their norms. When no confusion may arise we drop the subscript $A$.

For $s<0$ any real number, $\mathbf{H}^{s}(A)$ will denote the dual space of $\mathbf{H}_{0}^{-s}(A)$ 
endowed with the norm

$$
\|u\|_{s, A}=\sup _{\substack{v \in \mathbf{H}_{0}^{-s}(A) \\ v \neq 0}} \frac{\langle u, v\rangle}{\|v\|_{-s, A}},
$$

where $\langle\cdot, \cdot\rangle$ represents the duality pairing between $\mathbf{H}^{s}(A)$ and $\mathbf{H}_{0}^{-s}(A)$. We set

$$
\mathbf{H}(\operatorname{rot} ; A)=\left\{\boldsymbol{\delta} \in\left[\mathbf{L}^{2}(A)\right]^{2}, \operatorname{rot} \boldsymbol{\delta} \in \mathbf{L}^{2}(A)\right\},
$$

endowed with the graph norm

$$
\|\boldsymbol{\delta}\|_{\text {rot }, A}^{2}=\|\boldsymbol{\delta}\|_{0}^{2}+\|\operatorname{rot} \boldsymbol{\delta}\|_{0}^{2} .
$$

In addition, let $\tau$ be the unit vector tangential to $\partial A$ and oriented counterclockwise; then

$$
\mathbf{H}_{0}(\operatorname{rot} ; A)=\{\boldsymbol{\delta} \in \mathbf{H}(\operatorname{rot} ; A): \boldsymbol{\delta} \cdot \tau=0 \text { on } \partial A\} .
$$

The dual space of $\mathbf{H}_{0}(\operatorname{rot} ; A)$ is denoted by $\Gamma$ and has the following characterization:

$$
\boldsymbol{\Gamma}=\left(\mathbf{H}_{0}(\operatorname{rot} ; A)\right)^{\prime}=\left\{\chi \in\left[\mathbf{H}^{-1}(A)\right]^{2} ; \operatorname{div} \chi \in \mathbf{H}^{-1}(A)\right\},
$$

with the graph norm

$$
\|\chi\|_{\Gamma}^{2}=\|\chi\|_{-1}^{2}+\|\operatorname{div} \chi\|_{-1}^{2} .
$$

Let $s \in \mathscr{R}$; then for a triple $(\boldsymbol{\eta}, \boldsymbol{\delta}, q)$ with $\boldsymbol{\eta} \in\left[\mathbf{H}^{s}(A)\right]^{2}, \boldsymbol{\delta} \in \mathbf{H}(\operatorname{rot} ; A) \cap$ $\left[\mathbf{H}^{s-1}(A)\right]^{2}$, and $q \in \mathbf{H}^{s-1}(A)$ we introduce the following notation

$$
\|(\boldsymbol{\eta}, \boldsymbol{\delta}, q)\|_{s, A}^{2}=\|\boldsymbol{\eta}\|_{s, A}^{2}+\lambda^{-1} t^{2}\|\boldsymbol{\delta}\|_{s-1, A}^{2}+\|q\|_{s-1, A}^{2} .
$$

We use a circle above a function space to denote the subspace of elements with support contained in $A \subset \subset \Omega$. A circumflex above a function space denotes the subspace of elements with mean value zero. Vector-valued functions, operators, and spaces will be written in boldface.

Let $G_{0}$ and $G$, with $G_{0} \subset \subset G \subset \subset \Omega$ be arbitrary but fixed concentric sphere; then we make the following definition.

Definition 2.1. Let $\mathscr{P}_{h}$ be a linear operator defined on a Hilbert space $V$ with values in a finite-dimensional subspace $V_{h}$ of $V$. We say that $\mathscr{P}_{h}$ is local if there exists an $h_{0} \leq 1$, depending in general on $G_{0}$ and $G$, such that for all $h \in\left(0, h_{0}\right)$ and for every $u \in V$

$$
\operatorname{supp} u \subseteq \overline{G_{0}} \Rightarrow \operatorname{supp} \mathscr{P}_{h} u \subseteq \bar{G},
$$

where $\overline{G_{0}}$ and $\bar{G}$ stand for the closure of $G_{0}$ and $G$, respectively. Moreover, there exists a constant depending on $G_{0}, G$ such that

$$
\left\|\mathscr{P}_{h} u\right\|_{V, G_{0}} \leq C\|u\|_{V, G} .
$$

The letter $C$ denotes constants which are not necessarily the same in any two occurrences. The notation $C\left(a_{1}, \ldots, a_{n}\right)$ means that the constant $C$ depends on the $n$ known parameters $a_{1}, \ldots, a_{n}$. 
Finally, we recall the following standard differential operators for any scalar function $r$ and any vector-valued function $\mathbf{u}$ :

$$
\begin{array}{cc}
\operatorname{grad} r=\left(\begin{array}{c}
\frac{\partial r}{\partial x} \\
\frac{\partial r}{\partial y}
\end{array}\right), & \operatorname{rot} r=\left(\begin{array}{c}
\frac{\partial r}{\partial y} \\
-\frac{\partial r}{\partial x}
\end{array}\right), \\
\operatorname{div} \mathbf{u}=\frac{\partial \mathbf{u}_{1}}{\partial x}+\frac{\partial \mathbf{u}_{2}}{\partial y}, & \operatorname{rot} \mathbf{u}=\frac{\partial \mathbf{u}_{2}}{\partial x}-\frac{\partial \mathbf{u}_{1}}{\partial y} .
\end{array}
$$

\section{FORMULATION OF THE CONTINUOUS AND DISCRETE PROBLEMS}

Let us introduce the Euler equations for $\mathbf{J}_{t}$. The minimizers of (1.1) are then solutions of the following variational problem:

Problem 3.1. Find $(\boldsymbol{\theta}, w) \in\left[\mathbf{H}_{0}^{1}(\Omega)\right]^{2} \times \mathbf{H}_{0}^{1}(\Omega)$ such that

$$
\begin{gathered}
\mathbf{a}(\boldsymbol{\theta}, \boldsymbol{\eta})+(\boldsymbol{\gamma}, \operatorname{grad} v-\boldsymbol{\eta})=(F, v) \quad \forall(\boldsymbol{\eta}, v) \in\left[\mathbf{H}_{0}^{1}(\Omega)\right]^{2} \times \mathbf{H}_{0}^{1}(\Omega), \\
\gamma=\lambda t^{-2}(\operatorname{grad} w-\boldsymbol{\theta}),
\end{gathered}
$$

where the auxiliary variable is the shear strain.

For every $\boldsymbol{\theta}$ and $\boldsymbol{\eta}$ belonging to $\left[\mathrm{H}_{0}^{1}(\Omega)\right]^{2}$ the bilinear form a has the following expression:

$$
\begin{aligned}
\mathbf{a}(\boldsymbol{\theta}, \boldsymbol{\eta})=\frac{E}{12\left(1-\nu^{2}\right)} \int_{\Omega}\left[\left(\frac{\partial \boldsymbol{\theta}_{1}}{\partial x}\right.\right. & \left.+\nu \frac{\partial \boldsymbol{\theta}_{2}}{\partial y}\right) \frac{\partial \boldsymbol{\eta}_{1}}{\partial x}+\left(\nu \frac{\partial \boldsymbol{\theta}_{1}}{\partial x}+\frac{\partial \boldsymbol{\theta}_{2}}{\partial y}\right) \frac{\partial \boldsymbol{\eta}_{2}}{\partial y} \\
& \left.+\frac{1-\nu}{2}\left(\frac{\partial \boldsymbol{\theta}_{1}}{\partial y}+\frac{\partial \boldsymbol{\theta}_{2}}{\partial x}\right)\left(\frac{\partial \boldsymbol{\eta}_{1}}{\partial y}+\frac{\partial \boldsymbol{\eta}_{2}}{\partial x}\right)\right] d x d y .
\end{aligned}
$$

Then Korn's inequality

$$
\mathbf{a}(\boldsymbol{\eta}, \boldsymbol{\eta}) \geq K\|\boldsymbol{\eta}\|_{1} \quad \text { for every } \boldsymbol{\eta} \in\left[\mathbf{H}_{0}^{1}(\Omega)\right]^{2}
$$

and the theory of [3] give the existence and uniqueness of the solution and the following a priori estimate:

$$
\|\boldsymbol{\theta}\|_{1}+\|w\|_{1}+\|\gamma\|_{\Gamma}+t\|\gamma\|_{0} \leq C\|F\|_{-1} .
$$

In our analysis, we shall also make use of an equivalent formulation of (3.1) and (3.2), suggested by Brezzi and Fortin [7]. By applying the Helmholtz theorem, we decompose the shear strain vector in the following way:

$$
\gamma=\operatorname{grad} \psi+\operatorname{rot} p
$$

with $\psi \in \mathbf{H}_{0}^{1}(\Omega)$ and $p \in \widehat{\mathbf{H}}^{1}(\Omega)$; then the following variational formulation for Problem 3.1 can be derived:

Problem 3.2. Find $\psi \in \mathbf{H}_{0}^{1}(\boldsymbol{\Omega}),(\boldsymbol{\theta}, p) \in\left[\mathbf{H}_{0}^{1}(\Omega)\right]^{2} \times \widehat{\mathbf{H}}^{1}(\boldsymbol{\Omega})$, and $w \in \mathbf{H}_{0}^{1}(\boldsymbol{\Omega})$ such that

$$
\begin{gathered}
(\operatorname{grad} \psi, \operatorname{grad} \varphi)=(F, \varphi) \quad \forall \varphi \in \mathbf{H}_{0}^{1}(\Omega), \\
\mathbf{a}(\boldsymbol{\theta}, \boldsymbol{\eta})-(p, \operatorname{rot} \boldsymbol{\eta})=(\operatorname{grad} \psi, \boldsymbol{\eta}) \quad \forall \boldsymbol{\eta} \in\left[\mathbf{H}_{0}^{1}(\Omega)\right]^{2}, \\
(\boldsymbol{\theta}, \operatorname{\operatorname {rot}} q)+\lambda^{-1} t^{2}(\operatorname{rot} p, \operatorname{rot} q)=0 \quad \forall q \in \mathbf{H}^{1}(\Omega), \\
(\operatorname{grad} w, \operatorname{grad} v)=(\boldsymbol{\theta}, \operatorname{grad} v)+\lambda^{-1} t^{2}(F, v) \quad \forall v \in \mathbf{H}_{0}^{1}(\Omega) .
\end{gathered}
$$


Notice that this system of equations decouples in a natural way into a Poisson problem, a perturbation of a Stokes-like problem, and another Poisson problem. Hence, the analysis can be carried out sequentially. With the help of this formulation, the following a priori estimate can be derived uniformly with respect to $t$ (see for example [7]):

$$
\|\boldsymbol{\theta}\|_{2}+\|w\|_{2}+\|\psi\|_{2}+\|p\|_{1}+\lambda^{-1 / 2} t\|p\|_{2} \leq C\|F\|_{0} .
$$

When the regularity of $F$ increases, the solutions of the two Poisson equations become smoother, while the $\mathbf{H}^{s}$-norm of the solution of the Stokes-like problem is not uniformly bounded with respect to $t$ for any $s$. In [1], Arnold and Falk analyzed the structure of the boundary layer and proved the following best a priori estimate for $F \in \mathbf{H}^{1}(\Omega)$ :

$$
\|\boldsymbol{\theta}\|_{5 / 2}+\|p\|_{3 / 2}+\lambda^{-1 / 2} t\|p\|_{5 / 2} \leq C .
$$

On the other hand, at a positive distance from $\partial \Omega$ the boundary layer does not limit the regularity of $\theta$ and $p$. Thus, for $\Omega_{0} \subset \subset \Omega_{1} \subset \subset \Omega$ the following interior bound holds:

(3.13)

$$
\|\boldsymbol{\theta}\|_{s+2, \Omega_{0}}+\|w\|_{s+2, \Omega_{0}}+\|\psi\|_{s+2, \Omega_{0}}+\|p\|_{s+1, \Omega_{0}}+\lambda^{-1 / 2} t\|p\|_{s+2, \Omega_{0}} \leq C\|F\|_{s, \Omega_{1}} .
$$

We describe now the finite element approximation of (3.1), (3.2) that we shall consider (see [6]).

We assume henceforth that the domain $\Omega$ is decomposed by a regular and quasi-uniform mesh $\mathscr{T}_{h}$. As usual, we denote by $h$ the diameter of the largest element.

Let $\boldsymbol{\Theta}_{h}, W_{h}$, and $\boldsymbol{\Gamma}_{h}$ be finite element subspaces of $\left[\mathbf{H}_{0}^{1}(\Omega)\right]^{2}, \mathbf{H}_{0}^{1}(\Omega)$, and $\mathbf{H}_{0}(\operatorname{rot} ; \Omega)$, respectively, and let $\mathbf{R}$ be a linear operator with values in $\boldsymbol{\Gamma}_{h}$, related by the following conditions:

$$
\begin{gathered}
\mathbf{R} \Theta_{h} \subseteq \Gamma_{h}, \\
\operatorname{grad} W_{h} \subset \boldsymbol{\Gamma}_{h}, \\
(\mathbf{R}-\mathbf{I})\left(\operatorname{grad} W_{h}\right)=\{0\} .
\end{gathered}
$$

Assume furthermore that there exists another finite element space $Q_{h}$ such that

$$
\operatorname{rot} \Gamma_{h} \subseteq Q_{h},
$$

the pair $\left(\Theta_{h}, Q_{h}\right)$ is good for the Stokes problem,

$$
\operatorname{rot} \mathbf{R} \boldsymbol{\eta}=P_{0} \operatorname{rot} \boldsymbol{\eta}, \quad \text { for every } \boldsymbol{\eta} \in\left[\mathbf{H}_{0}^{1}(\Omega)\right]^{2}
$$

with $P_{0}: \mathbf{L}^{2}(\Omega) \mapsto Q_{h}$ denoting the $\mathbf{L}^{2}$-projection,

$$
\text { if } \boldsymbol{\delta}_{h} \in \boldsymbol{\Gamma}_{h} \text { is such that } \operatorname{rot} \boldsymbol{\delta}_{h}=0 \text {, then } \boldsymbol{\delta}_{h} \in \operatorname{grad} W_{h} \text {. }
$$

Some comments are in order about these conditions. Condition (3.18) means, more precisely, that there exists a linear operator $\Pi:\left[\mathbf{H}_{0}^{1}(\Omega)\right]^{2} \mapsto \boldsymbol{\Theta}_{h}$ such that for every $\boldsymbol{\eta} \in\left[\mathbf{H}_{0}^{1}(\Omega)\right]^{2}$

$$
\begin{gathered}
(\operatorname{rot}(\boldsymbol{\eta}-\Pi \boldsymbol{\eta}), q)=0 \quad \forall q \in Q_{h} \\
\|\Pi \boldsymbol{\eta}\|_{1} \leq C\|\boldsymbol{\eta}\|_{1} .
\end{gathered}
$$


The condition (3.19) is nothing but the commuting diagram property for elliptic problems. As a consequence of it, for every $\delta \in\left[\mathbf{H}_{0}^{1}(\Omega)\right]^{2}$ one has

$$
\|\mathbf{R} \boldsymbol{\delta}\|_{\text {rot }} \leq C\|\boldsymbol{\delta}\|_{\text {rot }} \text {. }
$$

Notice that the same space $Q_{h}$ is used to approximate both the pressure in the Stokes problem and the scalar field in the elliptic problem. Hence, it is preferable to consider elements for the Stokes problem with discontinuous pressure. Examples of spaces satisfying (3.14)-(3.20) are reported at the end of this section (we refer also to [6]).

The Euler equations of (1.2) can be written as follows:

Problem 3.3. Find $\left(\boldsymbol{\theta}_{h}, w_{h}\right) \in \boldsymbol{\theta}_{h} \times W_{h}$ such that

$$
\begin{gathered}
\mathbf{a}\left(\boldsymbol{\theta}_{h}, \boldsymbol{\eta}\right)+\left(\gamma_{h}, \operatorname{grad} v-\mathbf{R} \boldsymbol{\eta}\right)=(F, v) \quad \forall(\boldsymbol{\eta}, v) \in \boldsymbol{\Theta}_{h} \times W_{h}, \\
\gamma_{h}=\lambda t^{-2}\left(\operatorname{grad} w_{h}-\mathbf{R} \boldsymbol{\theta}_{h}\right) .
\end{gathered}
$$

In view of the properties listed above for the finite element spaces, we can introduce a decomposition principle similar to (3.6):

$$
\gamma_{h}=\operatorname{grad} \psi_{h}+\boldsymbol{\alpha}_{h}
$$

with $\psi_{h} \in W_{h}$ and $\left(\boldsymbol{\alpha}_{h}, p_{h}\right) \in \Gamma_{h} \times \widehat{Q}_{h}$ such that

$$
\left(\boldsymbol{\alpha}_{h}, \boldsymbol{\delta}\right)=\left(p_{h}, \operatorname{rot} \boldsymbol{\delta}\right) \quad \forall \boldsymbol{\delta} \in \boldsymbol{\Gamma}_{h} .
$$

We observe that (3.27) means that $\alpha_{h}=$ "rot ${ }_{h} p_{h}$ for an appropriate discretization of rot.

Then Problem 3.3 is equivalent to the following set of equations (see for example [8]):

Problem 3.4. Find $\psi_{h} \in W_{h}, \boldsymbol{\theta}_{h} \in \Theta_{h}, p_{h} \in \widehat{Q}_{h}, \boldsymbol{\alpha}_{h} \in \Gamma_{h}$, and $w_{h} \in W_{h}$ such that

$$
\begin{gathered}
\left(\operatorname{grad} \psi_{h}, \operatorname{grad} \varphi\right)=(F, \varphi) \quad \forall \varphi \in W_{h}, \\
\mathbf{a}\left(\boldsymbol{\theta}_{h}, \boldsymbol{\eta}\right)-\left(p_{h}, \operatorname{rot} \boldsymbol{\eta}\right)=\left(\operatorname{grad} \psi_{h}, \mathbf{R} \boldsymbol{\eta}\right) \quad \forall \boldsymbol{\eta} \in \boldsymbol{\Theta}_{h}, \\
\left(\operatorname{rot} \boldsymbol{\theta}_{h}, q\right)+\lambda^{-1} t^{2}\left(\operatorname{rot} \boldsymbol{\alpha}_{h}, q\right)=0 \quad \forall q \in Q_{h}, \\
\left(\boldsymbol{\alpha}_{h}, \boldsymbol{\delta}\right)-\left(p_{h}, \operatorname{rot} \boldsymbol{\delta}\right)=0 \quad \forall \boldsymbol{\delta} \in \boldsymbol{\Gamma}_{h}, \\
\left(\operatorname{grad} w_{h}, \operatorname{grad} v\right)=\left(\mathbf{R} \boldsymbol{\theta}_{h}, \operatorname{grad} v\right)+\lambda^{-1} t^{2}(F, v) \quad \forall v \in W_{h} .
\end{gathered}
$$

As in the continuous case, this set of equations can be solved sequentially. First one gets $\psi_{h}$ as a conforming approximation of the solution of an elliptic problem. Then $\left(\boldsymbol{\theta}_{h}, p_{h}, \boldsymbol{\alpha}_{h}\right)$ can be calculated by (3.29)-(3.31). Notice that (3.29), (3.30) is, for $t=0$, a Stokes-like problem discretized using the pair $\left(\Theta_{h}, Q_{h}\right)$. On the other hand, for $t \neq 0,(3.30),(3.31)$ is a mixed discretization, by means of the pair $\left(\boldsymbol{\Gamma}_{h}, Q_{h}\right)$, of a Neumann problem, with datum $\operatorname{rot} \boldsymbol{\theta}_{h}$. At the end, (3.32) is again the discretization of an elliptic problem by conforming elements.

In order to get the interior estimates, we shall analyze this system; hence it is better to write here the continuous problem corresponding to Problem 3.4. For this we introduce in Problem 3.2 the mixed formulation of (3.9), setting $\boldsymbol{\alpha}=\operatorname{rot} p$. Then we have 
Problem 3.5. Find $\psi \in \mathbf{H}_{0}^{1}(\Omega), \boldsymbol{\theta} \in\left[\mathbf{H}_{0}^{1}(\Omega)\right]^{2}, p \in \widehat{\mathbf{L}}^{2}(\Omega), \boldsymbol{\alpha} \in \mathbf{H}_{0}(\operatorname{rot} ; \Omega)$, and $w \in \mathbf{H}_{0}^{1}(\Omega)$ such that

$$
\begin{gathered}
(\operatorname{grad} \psi, \operatorname{grad} \varphi)=(F, \varphi) \quad \forall \varphi \in \mathbf{H}_{0}^{1}(\Omega), \\
\mathbf{a}(\boldsymbol{\theta}, \boldsymbol{\eta})-(p, \operatorname{rot} \boldsymbol{\eta})=(\operatorname{grad} \psi, \boldsymbol{\eta}) \quad \forall \boldsymbol{\eta} \in\left[\mathbf{H}_{0}^{1}(\Omega)\right]^{2}, \\
(\operatorname{rot} \boldsymbol{\theta}, q)+\lambda^{-1} t^{2}(\operatorname{rot} \boldsymbol{\alpha}, q)=0 \quad \forall q \in \mathbf{L}^{2}(\Omega), \\
(\boldsymbol{\alpha}, \boldsymbol{\delta})-(p, \operatorname{rot} \boldsymbol{\delta})=0 \quad \forall \boldsymbol{\delta} \in \mathbf{H}_{0}(\operatorname{rot} ; \Omega), \\
(\operatorname{grad} w, \operatorname{grad} v)=(\boldsymbol{\theta}, \operatorname{grad} v)+\lambda^{-1} t^{2}(F, v) \quad \forall v \in \mathbf{H}_{0}^{1}(\Omega) .
\end{gathered}
$$

3.1. Examples of finite element spaces. We present now some families of Reissner-Mindlin elements which satisfy the properties (3.14)-(3.20) (see [6] and [8]).

For the sake of simplicity we assume that $\Omega$ is a polygon. This does not imply a loss of generality.

We denote by $\mathscr{L}_{k}$ the set of the polynomials of degree $\leq k$.

Triangular family. Let $\Omega$ be decomposed into triangles $T$ and for any integer $k \geq 2$ define

$$
\begin{aligned}
& W_{h}=\left\{v \in \mathbf{H}_{0}^{1}(\Omega)|v|_{T} \in \mathscr{L}_{k}(T), T \in \mathscr{T}_{h}\right\}, \\
& \boldsymbol{\Theta}_{h}=\left\{\boldsymbol{\eta} \in\left[\mathbf{H}_{0}^{1}(\Omega)\right]^{2}|\boldsymbol{\eta}|_{T} \in\left[S_{k}(T)\right]^{2}, T \in \mathscr{T}_{h}\right\}
\end{aligned}
$$

with

$$
S_{k}(T)=\left\{\begin{array}{l}
\mathscr{L}_{k}(T) \text { for } k \geq 4, \\
\left\{v \in \mathscr{L}_{k+1}(T)|v|_{e} \in \mathscr{L}_{k}(e) \text { on each edge } e \text { of } T\right\} \quad \text { for } k=2,3 ;
\end{array}\right.
$$

then

(3.40) $\boldsymbol{\Gamma}_{h}=\left\{\boldsymbol{\delta} \in \mathbf{H}_{0}(\operatorname{rot} ; \Omega)|\boldsymbol{\delta}|_{T} \in\left[\mathscr{L}_{k-1}(T)\right]^{2} \oplus(x, y)^{\perp} \mathscr{L}_{k-1}(T), \quad T \in \mathscr{T}_{h}\right\}$.

$\Gamma_{h}$ is a kind of "rotated Raviart-Thomas" space, and it satisfies the commuting diagram property (3.19) for

$$
Q_{h}=\left\{q \in \mathbf{L}^{2}(\Omega)|q|_{T} \in \mathscr{L}_{k-1}(T), T \in \mathscr{T}_{h}\right\}
$$

with the reduction operator $\mathbf{R}$ defined locally by the following degrees of freedom: for $\boldsymbol{\eta}$ smooth in $T, \mathbf{R} \boldsymbol{\eta}$ satisfies

$$
\begin{gathered}
\int_{e}(\mathbf{R} \boldsymbol{\eta}-\boldsymbol{\eta}) \cdot \tau p_{k-1}(s) d s=0 \quad \forall e \text { edge of } T, \forall p_{k-1} \in \mathscr{L}_{k-1}(e), \\
\int_{T}(\mathbf{R} \boldsymbol{\eta}-\boldsymbol{\eta}) p_{k-2} d x d y=0 \quad \forall p_{k-2} \in\left[\mathscr{L}_{k-2}(T)\right]^{2} .
\end{gathered}
$$

We recall that these choices for the pair $\left(\boldsymbol{\Theta}_{h}, Q_{h}\right)$ correspond to well-known stable elements for the Stokes problem. We refer to [8] and the references quoted therein for the construction of the operator $\Pi$ satisfying (3.21)-(3.22).

Other triangular families can be obtained for $k=2,3$, substituting in $\Gamma_{h}$ the Raviart-Thomas space with the Brezzi-Douglas-Fortin-Marini one and consequently $\mathscr{L}_{k}$ by $S_{k}$ in $W_{h}$. 
Rectangular family. Let $\Omega$ be decomposed into rectangles $R$, and for $k=2,3$ define

$$
\begin{aligned}
& W_{h}=\left\{v \in \mathbf{H}_{0}^{1}(\Omega)|v|_{R} \in Q_{k} \cap \mathscr{L}_{k+1}, \quad R \in \mathscr{T}_{h}\right\}, \\
& \boldsymbol{\Theta}_{h}=\left\{\boldsymbol{\eta} \in\left[\mathbf{H}_{0}^{1}(\Omega)\right]^{2}|\boldsymbol{\eta}|_{R} \in\left[Q_{k}\right]^{2}, R \in \mathscr{T}_{h}\right\}, \\
& \boldsymbol{\Gamma}_{h}=\left\{\boldsymbol{\delta} \in \mathbf{H}_{0}(\operatorname{rot} ; \Omega)|\boldsymbol{\delta}|_{R} \in B D F M_{k}, R \in \mathscr{T}_{h}\right\} .
\end{aligned}
$$

$\Gamma_{h}$ is now a kind of "rotated Brezzi-Douglas-Fortin-Marini" space defined by

$$
B D F M_{k}=\left\{\boldsymbol{\delta} \mid \delta_{1} \in \mathscr{L}_{k} \backslash\left\{x^{k}\right\}, \delta_{2} \in \mathscr{L}_{k} \backslash\left\{y^{k}\right\}\right\} .
$$

It is known that $\Gamma_{h}$ satisfies the commuting diagram property (3.19) for

$$
Q_{h}=\left\{q \in \mathbf{L}^{2}(\Omega)|q|_{R} \in \mathscr{L}_{k-1}(R), R \in \mathscr{T}_{h}\right\}
$$

with the reduction operator $\mathbf{R}$ defined locally by the following degrees of freedom: for $\boldsymbol{\eta}$ smooth in $R, \mathbf{R} \boldsymbol{\eta}$ satisfies

$$
\begin{gathered}
\int_{e}(\mathbf{R} \boldsymbol{\eta}-\boldsymbol{\eta}) \cdot \tau p_{k-1}(s) d s=0 \quad \forall e \text { edge of } R, \forall p_{k-1} \in \mathscr{L}_{k-1}(e), \\
\int_{R}(\mathbf{R} \boldsymbol{\eta}-\boldsymbol{\eta}) p_{k-2} d x d y=0 \quad \forall p_{k-2} \in\left[\mathscr{L}_{k-2}(R)\right]^{2} .
\end{gathered}
$$

We refer to [6] for the proof that these spaces satisfy the properties listed above; we only mention that these choices for $\left(\boldsymbol{\Theta}_{h}, Q_{h}\right)$, for $k=2,3$, are stable for the Stokes problem (see for example [8]).

We end this subsection by stating the approximation properties of the finite element spaces presented:

$$
\begin{aligned}
& \|\boldsymbol{\eta}-\Pi \boldsymbol{\eta}\|_{l} \leq C h^{s-l}\|\boldsymbol{\eta}\|_{s} \\
& \forall \boldsymbol{\eta} \in\left[\mathbf{H}^{s}(\Omega) \cap \mathbf{H}_{0}^{1}(\Omega)\right]^{2}, \quad l=0,1, \quad l \leq s \leq k+1, \\
& \|\boldsymbol{\delta}-\mathbf{R} \boldsymbol{\delta}\|_{0}+h\|\operatorname{rot}(\boldsymbol{\delta}-\mathbf{R} \boldsymbol{\delta})\|_{0} \leq C h^{s}\|\boldsymbol{\delta}\|_{s} \\
& \forall \boldsymbol{\delta} \in\left[\mathbf{H}^{s}(\Omega)\right]^{2} \cap \mathbf{H}_{0}(\operatorname{rot} ; \Omega), \quad 1 \leq s \leq k, \\
& \left\|v-P_{0} v\right\|_{0} \leq C h^{s}\|v\|_{s} \quad \forall v \in \mathbf{H}^{s}(\Omega), \quad 0 \leq s \leq k, \\
& \left\|v-P_{1} v\right\|_{l} \leq C h^{s-l}\|v\|_{s} \\
& \forall v \in \mathbf{H}^{s}(\Omega) \cap \mathbf{H}_{0}^{1}(\Omega), \quad l=0,1,0 \leq s \leq k+1 .
\end{aligned}
$$

Here, $P_{1}$ is an interpolation operator constructed locally via a local averaging process such as that in [8] or [16]. Such an operator is often employed to construct $\Pi$ locally (see Proposition 2.9 of [8] and the examples quoted in Chapter VI). The operator $P_{1}$ in [16] turns out to be more convenient in the present setting because it preserves boundary conditions and is a projection operator, i.e., $P_{1} v=v$ for all $v \in \mathscr{L}_{k}$.

From the previous approximation properties the following error estimates can be derived (see [8] and [9]):

$$
\begin{gathered}
\left\|\boldsymbol{\theta}-\boldsymbol{\theta}_{h}\right\|_{1}+\left\|w-w_{h}\right\|_{1}+\left\|\psi-\psi_{h}\right\|_{1}+\left\|p-p_{h}\right\|_{0}+\lambda^{-1 / 2} t\left\|\boldsymbol{\alpha}-\boldsymbol{\alpha}_{h}\right\|_{0} \\
\leq C h^{s}\left(\|\boldsymbol{\theta}\|_{s+1}+\|w\|_{s+1}+\|\psi\|_{s+1}+\|p\|_{s}+\lambda^{-1 / 2} t\|p\|_{s+1}\right),
\end{gathered}
$$

with $s \leq \min (k, 3 / 2)$. 


\section{LOCAL ABSTRACT ASSUMPTIONS AND PRELIMINARIES}

Let $\Omega_{1}$ be a regular bounded subset of $\Omega$. We denote by $\boldsymbol{\theta}_{h}\left(\Omega_{1}\right)$ the finitedimensional subspace of $\left[\mathbf{H}^{1}\left(\Omega_{1}\right)\right]^{2}$ built up by the same piecewise polynomial functions as $\boldsymbol{\theta}_{h}$ (clearly without boundary constraints on $\partial \Omega_{1}$ ). For $\Omega_{0} \subset \Omega_{1}$ we define

$$
\stackrel{\circ}{\boldsymbol{\Theta}}_{h}\left(\Omega_{0}\right)=\left\{\boldsymbol{\eta} \in \Theta_{h}\left(\Omega_{1}\right) \mid \operatorname{supp} \boldsymbol{\eta} \subseteq \overline{\Omega_{0}}\right\} .
$$

Analogous meaning will have the notations $W_{h}\left(\Omega_{1}\right), \stackrel{\circ}{W}_{h}\left(\Omega_{0}\right), \Gamma_{h}\left(\Omega_{1}\right), \stackrel{\circ}{\Gamma}_{h}\left(\Omega_{0}\right)$, and $Q_{h}\left(\Omega_{1}\right), \stackrel{\circ}{Q}{ }_{h}\left(\Omega_{0}\right)$.

We make the following local approximability assumptions concerning $\Theta_{h}\left(\Omega_{1}\right), W_{h}\left(\Omega_{1}\right), \Gamma_{h}\left(\Omega_{1}\right)$, and $Q_{h}\left(\Omega_{1}\right)$. Let $G_{0}$ and $G$, with $G_{0} \subset \subset G \subset \subset$ $\Omega_{1}$, be arbitrary but fixed concentric spheres. We shall denote by $G_{h}$ a union of elements of $\mathscr{T}_{h}$ which contains $G_{0}$ and is contained in $G$.

There exists an $h_{0} \leq 1$, depending in general on $G_{0}$ and $G$, such that for all $h \in\left(0, h_{0}\right)$ there holds:

A1. The linear operators $\Pi, \mathbf{R}, P_{0}$, and $P_{1}$ are local (see Definition 2.1) and

$$
\begin{aligned}
& \|\boldsymbol{\eta}-\Pi \boldsymbol{\eta}\|_{l, G} \leq C h^{s-l}\|\boldsymbol{\eta}\|_{s, G_{0}} \\
& \forall \boldsymbol{\eta} \in\left[\stackrel{\circ}{\mathbf{H}}^{s}\left(G_{0}\right)\right]^{2}, l=0,1, l \leq s \leq k+1, \\
& \|\boldsymbol{\delta}-\mathbf{R} \boldsymbol{\delta}\|_{0, G}+h\|\operatorname{rot}(\boldsymbol{\delta}-\mathbf{R} \boldsymbol{\delta})\|_{0, G} \leq C h^{s}\|\boldsymbol{\delta}\|_{s, G_{0}} \\
& \forall \boldsymbol{\delta} \in\left[\stackrel{\circ}{\mathbf{H}^{s}}\left(G_{0}\right)\right]^{2}, \quad 1 \leq s \leq k, \\
& \left\|v-P_{0} v\right\|_{0, G} \leq C h^{s}\|v\|_{s, G_{0}} \quad \forall v \in \stackrel{\circ}{\mathbf{H}}^{s}\left(G_{0}\right), \quad 0 \leq s \leq k, \\
& \left\|v-P_{1} v\right\|_{l, G} \leq C h^{s-l}\|v\|_{s, G_{0}} \\
& \forall v \in \stackrel{\circ}{\mathbf{H}}^{s}\left(G_{0}\right), \quad l=0,1,0 \leq s \leq k+1 .
\end{aligned}
$$

Remark 4.1. In particular, if $\omega \in \mathbf{C}_{0}^{\infty}\left(G_{0}\right)$ and $\boldsymbol{\eta} \in\left[\mathbf{H}^{s}(G)\right]^{2}$, then $\omega \boldsymbol{\eta} \in$ $\left[\stackrel{\circ}{\mathbf{H}}^{s}\left(G_{0}\right)\right]^{2}$, and we can apply A1 relative to $\Pi$, so that $\Pi(\omega \eta) \in{\stackrel{\circ}{\Theta_{h}}}_{h}(G)$ and from $(4.1)$

$$
\|\omega \boldsymbol{\eta}-\Pi(\omega \boldsymbol{\eta})\|_{l, G} \leq C h^{s-l}\|\boldsymbol{\eta}\|_{s, G_{0}}, \quad l=0,1, l \leq s \leq k+1 .
$$

Analogous remarks hold for the operators $\mathbf{R}, P_{0}$, and $P_{1}$.

A2. Let $\omega \in \mathbf{C}_{0}^{\infty}\left(G_{0}\right)$; then there exists $G_{h}$ such that $G_{0} \subset \subset G_{h} \subset \subset G$ and

$$
\begin{array}{ll}
\text { (4.5) } & \left\|\omega \boldsymbol{\eta}_{h}-\Pi\left(\omega \boldsymbol{\eta}_{h}\right)\right\|_{1, G} \leq C h\left\|\boldsymbol{\eta}_{h}\right\|_{1, G_{h}} \quad \forall \boldsymbol{\eta}_{h} \in \boldsymbol{\Theta}_{h}\left(\Omega_{1}\right), \\
\text { (4.6) } & \left\|\omega \boldsymbol{\delta}_{h}-\mathbf{R}\left(\omega \boldsymbol{\delta}_{h}\right)\right\|_{0, G} \leq C h\left\|\boldsymbol{\delta}_{h}\right\|_{0, G_{h}} \quad \forall \boldsymbol{\delta}_{h} \in \Gamma_{h}\left(\Omega_{1}\right), \\
\text { (4.7) } & \left\|\omega q_{h}-P_{0}\left(\omega q_{h}\right)\right\|_{0, G} \leq C h\left\|q_{h}\right\|_{0, G_{h}} \quad \forall q_{h} \in Q_{h}\left(\Omega_{1}\right), \\
\text { (4.8) } & \left\|\omega v_{h}-P_{1}\left(\omega v_{h}\right)\right\|_{l, G} \leq C h^{2-l}\left\|v_{h}\right\|_{1, G_{h}} \quad \forall v_{h} \in W_{h}\left(\Omega_{1}\right), \quad l=0,1 .
\end{array}
$$

A3. Let $G_{h}$ be as in A2; then for all $\delta_{h} \in \Gamma_{h}\left(\Omega_{1}\right)$ there holds

$$
\left\|\operatorname{rot}\left(\omega \boldsymbol{\delta}_{h}\right)-\operatorname{rot} \mathbf{R}\left(\omega \boldsymbol{\delta}_{h}\right)\right\|_{0, G} \leq C h\left\|\boldsymbol{\delta}_{h}\right\|_{0, G_{h}}+C h\left\|\operatorname{rot} \boldsymbol{\delta}_{h}\right\|_{0, G_{h}} .
$$


The Mindlin-Reissner elements introduced in the previous section enjoy the local approximability assumptions A1-A3:

It is easy to see that $\mathrm{A} 1$ holds, since the operators $\Pi, \mathbf{R}, P_{0}$, and $P_{1}$ are defined locally, possibly through a local averaging process (see [16]). A2 is more delicate, and we refer to [11] for the technical details of its proof in the case of the triangular family with $k=2$. For the proof of $\mathrm{A} 2$ with other choices of spaces see also [4], [10], and [12], [15] in an analogous context.

We now verify A3 for the triangular family with $k=2$; the proof is similar for $k>2$. The assumption (3.19) in conjunction with Remark 4.1 implies the following bound:

$$
\left\|\operatorname{rot}\left(\omega \boldsymbol{\delta}_{h}\right)-\operatorname{rot} \mathbf{R}\left(\omega \boldsymbol{\delta}_{h}\right)\right\|_{0, G} \leq C h^{2}\left(\sum_{T}\left\|\operatorname{rot}\left(\omega \boldsymbol{\delta}_{h}\right)\right\|_{2, T}^{2}\right)^{1 / 2}
$$

where the sum is taken over those triangles of $\mathscr{T}_{h}$ whose union $G_{h}$ contains the support of $\omega$. It remains to evaluate the $\mathbf{L}^{2}$-norm of the second derivatives of $\operatorname{rot}\left(\omega \delta_{h}\right)$. Recalling that $\operatorname{rot}\left(\omega \delta_{h}\right)=\operatorname{rot} \omega \cdot \delta_{h}+\omega \operatorname{rot} \delta_{h}$, we get from the Leibniz rule

$$
\left\|\operatorname{rot}\left(\omega \boldsymbol{\delta}_{h}\right)\right\|_{2, T}^{2} \leq C \sum_{j=0}^{2}\left(\left\|D^{j}(\operatorname{rot} \omega) D^{2-j} \boldsymbol{\delta}_{h}\right\|_{0, T}^{2}+\left\|D^{j}(\omega) D^{2-j}\left(\operatorname{rot} \boldsymbol{\delta}_{h}\right)\right\|_{0, T}^{2}\right) .
$$

Then, since $\omega \in \mathbf{C}_{0}^{\infty}\left(G_{0}\right)$ and $\operatorname{rot} \delta_{h \mid T} \in P_{1}$, we obtain by the inverse inequality

$$
\left\|\operatorname{rot}\left(\omega \boldsymbol{\delta}_{h}\right)\right\|_{2, T}^{2} \leq C h^{-2}\left(\left\|\delta_{h}\right\|_{0, T}^{2}+\left\|\operatorname{rot} \delta_{h}\right\|_{0, T}^{2}\right)+C\left\|D^{2} \delta_{h}\right\|_{0, T}^{2} .
$$

To bound the last term, we recall that the elements of $\boldsymbol{\Gamma}_{h}$ have on each triangle the following representation:

$$
\begin{aligned}
& \delta_{1 h}=a_{1}+b_{1} x+c_{1} y+y(d x+e y), \\
& \delta_{2 h}=a_{2}+b_{2} x+c_{2} y-x(d x+e y),
\end{aligned}
$$

so that the derivatives $\partial^{2} \delta_{1 h} / \partial x^{2}, \partial^{2} \delta_{2 h} / \partial y^{2}, 2 \partial^{2} \delta_{1 h} / \partial x \partial y+\partial^{2} \delta_{2 h} / \partial x^{2}$, $\partial^{2} \delta_{1 h} / \partial y^{2}+2 \partial^{2} \delta_{2 h} / \partial x \partial y$ all vanish. This implies that we can write each second derivative of the components of $\delta_{h}$ in terms of a first derivative of rot $\delta_{h}$, so that we have the following estimate:

$$
\left\|D^{2} \delta_{h}\right\|_{0, T} \leq C\left\|D \operatorname{rot} \delta_{h}\right\|_{0, T} \leq C h^{-1}\left\|\operatorname{rot} \delta_{h}\right\|_{0, T},
$$

which inserted in (4.11) and back in (4.10) gives (4.9).

Remark 4.2. The assumption A3 does not hold for rectangular families. In fact, suppose that the sphere $G_{0}$ contains strictly the rectangle $R=(-h, h) \times(0, h)$, divided into two elements. Next take

$$
\boldsymbol{\delta}_{h}=\frac{1}{h^{2}}(y(y-h) \operatorname{sign} x,(2 y-h)(x \operatorname{sign} x-h))
$$

in $R$ and $\boldsymbol{\delta}_{h}=(0,0)$ otherwise. Moreover, let $\omega \in \mathbf{C}_{0}^{\infty}\left(G_{0}\right)$ be such that $\omega(x, y)=x$ for $(x, y) \in R$ and calculate $\operatorname{rot} \mathbf{R}\left(\omega \delta_{h}\right)$. Then it is easy to see that $\left\|\operatorname{rot}\left(\omega \delta_{h}\right)-\operatorname{rot} \mathbf{R}\left(\omega \boldsymbol{\delta}_{h}\right)\right\|_{0, R}=O(h), h\left\|\boldsymbol{\delta}_{h}\right\|_{0, R}=O\left(h^{2}\right)$, and $\operatorname{rot} \boldsymbol{\delta}_{h}=0$.

We now state some technical lemmas, whose easy proofs are based on differentiation rules and integration by parts (see [11] for the details). 


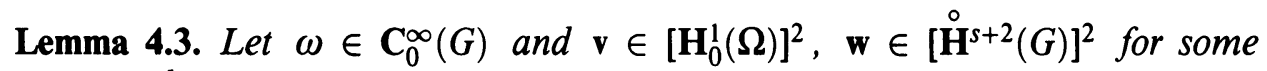
$s \geq-1$; then

$$
\mathbf{a}(\omega \mathbf{v}, \mathbf{w})=\mathbf{a}(\mathbf{v}, \omega \mathbf{w})-I(\omega \mathbf{v}, \mathbf{w})
$$

and

$$
I(\omega \mathbf{v}, \mathbf{w}) \leq C(\omega, G)\|\mathbf{v}\|_{-s-1, G}\|\mathbf{w}\|_{s+2, G}
$$

Lemma 4.4. Let $\omega \in \mathbf{C}_{0}^{\infty}(G), r \in \stackrel{\circ}{H}^{s+1}(G)$, and $\mathbf{v} \in\left[\mathbf{H}_{0}^{1}(\Omega)\right]^{2}$ for some $s \geq-1$; then

$$
(\operatorname{rot}(\omega \mathbf{v}), r)=(\omega r, \operatorname{rot} \mathbf{v})-((\operatorname{rot} \omega) r, \mathbf{v})
$$

with

$$
((\operatorname{rot} \omega) r, \mathbf{v}) \leq C(\omega, G)\|\mathbf{v}\|_{-s-1, G}\|r\|_{s+1, G} .
$$

Lemma 4.5. Let $\omega \in \mathbf{C}_{0}^{\infty}(G)$; then for every $q \in Q_{h}, \boldsymbol{\eta} \in \boldsymbol{\Theta}_{h}, \boldsymbol{\delta} \in \boldsymbol{\Gamma}_{h}$, the following equalities hold:

$$
\begin{aligned}
(q, \operatorname{rot} \Pi(\omega \boldsymbol{\eta}))= & (q,-\operatorname{rot} \omega \cdot \boldsymbol{\eta})+\left(\omega q-P_{0}(\omega q), \operatorname{rot} \boldsymbol{\eta}\right) \\
& +\left(P_{0}(\omega q), \operatorname{rot} \boldsymbol{\eta}\right) \\
(q, \operatorname{rot} \mathbf{R}(\omega \boldsymbol{\delta}))= & (q,-\operatorname{rot} \omega \cdot \boldsymbol{\delta})+\left(P_{0}(\omega q), \operatorname{rot} \boldsymbol{\delta}\right) .
\end{aligned}
$$

\section{AN AUXILIARY PROBLEM}

Consider the following auxiliary problem: find $(\mathbf{z}, r)$ with $\int_{\Omega} r=0$ that satisfy

$$
\begin{gathered}
\mathbf{A z}-\operatorname{rot} r=\mathbf{f} \text { in } \Omega, \\
-\operatorname{rot} \mathbf{z}+\lambda^{-1} t^{2} \Delta r=g+\lambda^{-1} t^{2} \operatorname{rot} \phi \quad \text { in } \Omega, \\
\mathbf{z}=0, \quad \frac{\partial r}{\partial n}=0 \quad \text { on } \partial \Omega,
\end{gathered}
$$

where $\mathbf{A}$ is the second-order operator associated with the bilinear form $\mathbf{a}$, that is,

$$
(\mathbf{A z}, \boldsymbol{\eta})=\mathbf{a}(\mathbf{z}, \boldsymbol{\eta}) \quad \forall \mathbf{z}, \boldsymbol{\eta} \in\left[\mathbf{H}_{0}^{1}(\Omega)\right]^{2} .
$$

If $\phi \neq 0$, we can introduce the additional variable $\chi$ which satisfies

$$
\chi=\operatorname{rot} r+\phi \quad \text { in } \Omega, \quad \chi \cdot \tau=0 \text { on } \partial \Omega .
$$

Substituting (5.4) in (5.2), we obtain the system

$$
\begin{gathered}
\mathbf{A z}-\operatorname{rot} r=\mathbf{f} \text { in } \Omega, \\
-\operatorname{rot} \mathbf{z}-\lambda^{-1} t^{2} \operatorname{rot} \chi=g \quad \text { in } \Omega, \\
\chi-\operatorname{rot} r=\phi \quad \text { in } \Omega, \\
\mathbf{z}=0, \quad \frac{\partial r}{\partial n}=0, \quad \chi \cdot \tau=0 \quad \text { on } \partial \Omega .
\end{gathered}
$$

In the following lemmas we state some a priori estimates for the solutions of (5.1) $-(5.3)$ and (5.5)-(5.8). 
Lemma 5.1. Let $\mathbf{f} \in\left[\mathrm{H}^{-1}(\Omega)\right]^{2}$ and $g \in \mathbf{L}^{2}(\Omega)$, with $\int_{\Omega} g=0$, be given and suppose that $\phi=0$. Then the solution of (5.1)-(5.3) satisfies the following a priori estimate uniformly with respect to $t$ :

$$
\|\mathbf{z}\|_{1, \Omega}+\|r\|_{0, \Omega}+\lambda^{-1 / 2} t\|r\|_{1, \Omega}+\lambda^{-1} t^{2}\|r\|_{2, \Omega} \leq C\left(\|\mathbf{f}\|_{-1, \Omega}+\|g\|_{0, \Omega}\right) .
$$

If $\mathbf{f} \in\left[\mathbf{L}^{2}(\Omega)\right]^{2}$ and $g \in \mathbf{H}_{0}^{1}(\Omega)$, then

$$
\|\mathbf{z}\|_{2, \Omega}+\|r\|_{1, \Omega}+\lambda^{-1 / 2} t\|r\|_{2, \Omega} \leq C\left(\|\mathbf{f}\|_{0, \Omega}+\|g\|_{1, \Omega}\right) .
$$

Lemma 5.2. Let $\mathbf{f} \in\left[\mathbf{H}^{2}(\Omega)\right]^{2}, g \in \mathbf{H}^{3}(\Omega)$, with $\int_{\Omega} g=0$, and $\boldsymbol{\phi}=0$; then there exists a positive constant $C$ depending on $\mathbf{f}, g, \Omega$ such that

$$
\|\mathbf{z}\|_{5 / 2, \Omega}+\|r\|_{3 / 2, \Omega}+\lambda^{-1 / 2} t\|r\|_{5 / 2, \Omega} \leq C .
$$

Lemma 5.3. If $\mathbf{f} \in\left[\stackrel{\circ}{\mathbf{H}}^{s}(\Omega)\right]^{2}, g \in \stackrel{\circ}{\mathbf{H}}^{s+1}(\Omega)$, with $\int_{\Omega} g=0$, and $\phi=0$, then for every open subset $\Omega^{\prime}$ such that $\Omega^{\prime} \subset \subset \Omega$, there holds

$$
\begin{aligned}
& \|\mathbf{z}\|_{s+2, \Omega^{\prime}}+\|r\|_{s+1, \Omega^{\prime}}+\lambda^{-1 / 2} t\|r\|_{s+2, \Omega^{\prime}}+\lambda^{-1} t^{2}\|r\|_{s+3, \Omega^{\prime}} \\
& \quad \leq C\left(\|\mathbf{f}\|_{s, \Omega}+\|g\|_{s+1, \Omega}\right) .
\end{aligned}
$$

The results of Lemmas 5.1-5.3 can be deduced from [7], [1] and by means of standard techniques for interior a priori estimates (see, for example [13]).

Lemma 5.4. Let $(\mathbf{z}, \chi, r)$ be the solution of (5.5)-(5.8) with $\mathbf{f}=g=0$ and $\phi \in\left[\mathbf{H}^{1}(\Omega)\right]^{2} ;$ then

$$
\|\mathbf{z}\|_{2, \Omega}+\|r\|_{1, \Omega}+\lambda^{-1 / 2} t\|r\|_{2, \Omega}+\lambda^{-1 / 2} t\|\chi\|_{1, \Omega} \leq C \lambda^{-1 / 2} t\|\phi\|_{1, \Omega} .
$$

If $\phi \in\left[\mathbf{H}^{2}\right]^{2}$, then

$$
\|\mathbf{z}\|_{5 / 2, \Omega}+\|r\|_{3 / 2, \Omega}+\lambda^{-1 / 2} t\|r\|_{5 / 2, \Omega}+\lambda^{-1 / 2} t\|\chi\|_{3 / 2, \Omega} \leq C \lambda^{-1 / 2} t .
$$

Proof. The inequality (5.13) is a consequence of (5.10) in this case. In fact since $\mathbf{A}$ is an elliptic operator with constant coefficients, we get from (5.5)

$$
\|\mathbf{z}\|_{2, \Omega} \leq C\|\mathbf{A z}\|_{0, \Omega} \leq C\|r\|_{1, \Omega} \leq C \lambda^{-1 / 2} t\|\phi\|_{1, \Omega} .
$$

Then it is easily seen that

$$
\|\chi\|_{1, \Omega} \leq C\|\phi\|_{1, \Omega} .
$$

The proof of $(5.14)$ is similar.

Lemma 5.5. If $\mathbf{f}=g=0$ and $\phi \in\left[\stackrel{\circ}{H}^{s}(\Omega)\right]^{2}$, then for every subset $\Omega^{\prime}$ such that $\Omega^{\prime} \subset \subset \Omega$, there holds

$$
\|\mathbf{z}\|_{s+1, \Omega^{\prime}}+\|r\|_{s, \Omega^{\prime}}+\lambda^{-1 / 2} t\|r\|_{s+1, \Omega^{\prime}}+\lambda^{-1} t^{2}\|r\|_{s+2, \Omega^{\prime}} \leq C \lambda^{-1} t^{2}\|\phi\|_{s+1, \Omega}
$$

and

$$
\|\mathbf{z}\|_{s+2, \Omega^{\prime}}+\lambda^{-1 / 2} t\|\chi\|_{s+1, \Omega^{\prime}} \leq C \lambda^{-1 / 2} t\|\phi\|_{s+1, \Omega} .
$$

Proof. The proof is analogous to the previous one. 


\section{THE MAIN RESULTS}

In this section we describe the main steps to achieve the interior error estimates, for both the physical variables, the rotation of fibers $\theta$, and the transverse displacement $w$. Let $\Omega_{0}$ and $\Omega_{1}$ be two open bounded subsets of $\Omega$ such that $\Omega_{0} \subset \subset \Omega_{1} \subset \subset \Omega$. Following [14], we are going to show that the approximation error in $\Omega_{0}$ can be bounded by two terms: the first has the best order of accuracy possible in $\Omega_{1}$, the second is the negative norm \|\|$_{-\mu, \Omega_{1}}$ of the error ( $\mu$ being an arbitrary nonnegative integer).

We proceed by analyzing the difference between the solutions of Problems 3.4 and 3.5. We thus split our analysis into three parts: first we consider the approximation of the Poisson equation (3.33) by (3.28), then we compare the discrete solution of the Stokes-like system (3.29)-(3.31) with the continuous solution of (3.34)-(3.36), and we evaluate the error for the Poisson equation (3.37) discretized by (3.32).

6.1. Interior error estimates for the first Poisson equation. We bound the norm $\left\|\psi-\psi_{h}\right\|_{1, \Omega_{0}}$. For the Poisson problem, estimates of such quantities are already available. Namely, from [14, Theorem 5.1], we have, if $\psi \in \mathbf{H}^{s}\left(\Omega_{1}\right)$,

$$
\left\|\psi-\psi_{h}\right\|_{1, \Omega_{0}} \leq C\left(h^{s}\|\psi\|_{s+1, \Omega_{1}}+\left\|\psi-\psi_{h}\right\|_{-\mu_{1}, \Omega_{1}}\right),
$$

with $0 \leq s \leq k$ and $\mu_{1}$ a nonnegative integer.

6.2. Interior error estimates for the Stokes-like problem. We now focus on the Stokes-like system. The first step is to prove a local version of the interior estimates, considering two concentric spheres $G_{0}$ and $G$, instead of $\Omega_{0}$ and $\Omega_{1}$. Next we apply such local estimates to a finite number of spheres which cover $\bar{\Omega}_{0}$. Therefore, we start by proving the following local error bound.

Theorem 6.1. Assume that the assumptions A1-A3 are verified. Let $G_{0} \subset \subset$ $G \subset \subset \Omega_{1}$ be concentric spheres; then we have

$$
\begin{aligned}
&\left\|\left(\boldsymbol{\theta}-\boldsymbol{\theta}_{h}, \boldsymbol{\alpha}-\boldsymbol{\alpha}_{h}, p-p_{h}\right)\right\|_{1, G_{0}} \\
& \leq C\left\|\psi-\psi_{h}\right\|_{1, G}+\left\|\omega \psi-P_{1}(\omega \psi)\right\|_{1, G} \\
&+\left\|\left(\omega \boldsymbol{\theta}-\Pi(\omega \boldsymbol{\theta}), \omega \boldsymbol{\alpha}-\mathbf{R}(\omega \boldsymbol{\alpha}), \omega p-P_{0}(\omega p)\right)\right\|_{1, G} \\
&\left.+\left\|\left(\boldsymbol{\theta}-\boldsymbol{\theta}_{h}, \boldsymbol{\alpha}-\boldsymbol{\alpha}_{h}, p-p_{h}\right)\right\|_{-\mu_{2}, G}+\sup _{\substack{\circ \\
\boldsymbol{\bullet}}} \frac{(\operatorname{grad}(\omega \psi), \boldsymbol{\eta}-\mathbf{R} \boldsymbol{\eta})}{\|\boldsymbol{\eta}\|_{1, G}}\right),
\end{aligned}
$$

where $\mu_{2}$ is a nonnegative integer and $\omega$ is a cutoff function defined as follows:

$$
\omega \in \mathbf{C}_{0}^{\infty}\left(G^{\prime}\right) \text { and } \omega \equiv 1 \text { on } G_{0}^{\prime} \text {, }
$$

with $G_{0}^{\prime}$ and $G^{\prime}$ such that $G_{0} \subset \subset G_{0}^{\prime} \subset \subset G^{\prime} \subset \subset G \subset \subset \Omega_{1}$.

Proof. We set

$$
\tilde{\boldsymbol{\theta}}=\omega \boldsymbol{\theta}, \quad \tilde{p}=\omega p, \quad \tilde{\boldsymbol{\alpha}}=\omega \boldsymbol{\alpha} .
$$

Then $\tilde{\boldsymbol{\theta}} \in\left[\stackrel{\circ}{\mathbf{H}}^{1}\left(G^{\prime}\right)\right]^{2}, \tilde{p} \in \stackrel{\circ}{\mathbf{L}}^{2}\left(G^{\prime}\right)$, and $\tilde{\boldsymbol{\alpha}} \in \stackrel{\circ}{\mathbf{H}}\left(\operatorname{rot} ; G^{\prime}\right)$, and owing to (3.34)(3.36) and Lemmas 4.3 and 4.4 , they satisfy 


$$
\begin{aligned}
\mathbf{a}(\tilde{\boldsymbol{\theta}}, \boldsymbol{\eta})-(\tilde{\boldsymbol{p}}, \operatorname{rot} \boldsymbol{\eta})= & (\operatorname{grad}(\omega \psi), \boldsymbol{\eta})-I(\omega \boldsymbol{\theta}, \boldsymbol{\eta}) \\
& -((\operatorname{rot} \omega) p+(\operatorname{grad} \omega) \psi, \boldsymbol{\eta}) \quad \forall \boldsymbol{\eta} \in\left[\stackrel{\circ}{\mathbf{H}}^{1}(G)\right]^{2},
\end{aligned}
$$

$(\operatorname{rot} \tilde{\boldsymbol{\theta}}, q)+\lambda^{-1} t^{2}(\operatorname{rot} \tilde{\boldsymbol{\alpha}}, q)=-\left(\operatorname{rot} \omega \cdot\left(\boldsymbol{\theta}+\lambda^{-1} t^{2} \boldsymbol{\alpha}\right), q\right) \quad \forall q \in \stackrel{\circ}{\mathbf{L}}^{2}(G)$,

Let $\tilde{\boldsymbol{\theta}}_{h} \in{\stackrel{\circ}{\boldsymbol{\Theta}_{h}}}_{h}(G), \widetilde{\boldsymbol{\alpha}_{h}} \in \stackrel{\circ}{\Gamma}_{h}(G), \widetilde{p_{h}} \in \stackrel{\circ}{Q}_{h}(G)$ with $\int_{G} \widetilde{p_{h}}=\int_{G} \tilde{p}$, be the unique solution of

$$
\begin{aligned}
\mathbf{a}(\tilde{\boldsymbol{\theta}}- & \left.\tilde{\boldsymbol{\theta}}_{h}, \boldsymbol{\eta}\right)-\left(\tilde{\boldsymbol{p}}-\widetilde{p_{h}}, \operatorname{rot} \boldsymbol{\eta}\right) \\
& =(\operatorname{grad}(\omega \psi), \boldsymbol{\eta})-\left(\operatorname{grad} P_{1}(\omega \psi), \mathbf{R} \boldsymbol{\eta}\right) \quad \forall \boldsymbol{\eta} \in{\stackrel{\circ}{\Theta_{h}}}_{(G),}
\end{aligned}
$$

$$
\begin{gathered}
\left(\operatorname{rot}\left(\tilde{\boldsymbol{\theta}}-\tilde{\boldsymbol{\theta}}_{h}\right), q\right)+\lambda^{-1} t^{2}\left(\operatorname{rot}\left(\tilde{\boldsymbol{\alpha}}-\widetilde{\boldsymbol{\alpha}_{h}}\right), q\right)=0 \quad \forall q \in{\stackrel{\circ}{Q_{h}}}_{(G),} \\
\left(\tilde{\boldsymbol{\alpha}}-\widetilde{\boldsymbol{\alpha}_{h}}, \boldsymbol{\delta}\right)-\left(\tilde{p}-\widetilde{p_{h}}, \operatorname{rot} \boldsymbol{\delta}\right)=0 \quad \forall \boldsymbol{\delta} \in \stackrel{\circ}{\boldsymbol{\Gamma}}_{h}(G) .
\end{gathered}
$$

We recall that $P_{1}(\omega \psi) \in \stackrel{\circ}{W}_{h}(G)$ in view of Remark 4.1. On the other hand, we have from Problems 3.4 and 3.5 the following error equations:

$$
\begin{aligned}
& \mathbf{a}\left(\boldsymbol{\theta}-\boldsymbol{\theta}_{h}, \boldsymbol{\eta}\right)-\left(p-p_{h}, \operatorname{rot} \boldsymbol{\eta}\right) \\
& \quad=(\operatorname{grad} \psi, \boldsymbol{\eta})-\left(\operatorname{grad} \psi_{h}, \mathbf{R} \boldsymbol{\eta}\right) \quad \forall \boldsymbol{\eta} \in \boldsymbol{\Theta}_{h}(\boldsymbol{\Omega}), \\
& \left(\operatorname{rot}\left(\boldsymbol{\theta}-\boldsymbol{\theta}_{h}\right), q\right)+\lambda^{-1} t^{2}\left(\operatorname{rot}\left(\boldsymbol{\alpha}-\boldsymbol{\alpha}_{h}\right), q\right)=0 \quad \forall q \in Q_{h}(\boldsymbol{\Omega}), \\
& \left(\boldsymbol{\alpha}-\boldsymbol{\alpha}_{h}, \boldsymbol{\delta}\right)-\left(p-p_{h}, \operatorname{rot} \boldsymbol{\delta}\right)=0 \quad \forall \boldsymbol{\delta} \in \boldsymbol{\Gamma}_{h}(\Omega) .
\end{aligned}
$$

We compare these two sets of error equations for all $\eta \in \stackrel{\circ}{\Theta}_{h}\left(G_{0}^{\prime}\right), q \in \stackrel{\circ}{Q}_{h}\left(G_{0}^{\prime}\right)$, and $\boldsymbol{\delta} \in \stackrel{\circ}{\Gamma}_{h}\left(G_{0}^{\prime}\right)$, taking into account that, from (6.3) and (6.4), $\boldsymbol{\theta}=\tilde{\boldsymbol{\theta}}, p=\tilde{p}$, and $\alpha=\tilde{\alpha}$ in $G_{0}^{\prime}$. Hence, subtracting (6.11)-(6.13) from (6.8)-(6.10), we arrive at

$$
\begin{gathered}
\mathbf{a}\left(\boldsymbol{\theta}_{h}-\tilde{\boldsymbol{\theta}}_{h}, \boldsymbol{\eta}\right)-\left(p_{h}-\widetilde{p_{h}}, \operatorname{rot} \boldsymbol{\eta}\right) \\
=\left(\operatorname{grad}\left(\psi_{h}-P_{1}(\omega \psi)\right), \mathbf{R} \boldsymbol{\eta}\right) \quad \forall \boldsymbol{\eta} \in{\stackrel{\circ}{\boldsymbol{\Theta}_{h}}}\left(G_{0}^{\prime}\right), \\
\left(\operatorname{rot}\left(\boldsymbol{\theta}_{h}-\tilde{\boldsymbol{\theta}}_{h}\right), q\right)+\lambda^{-1} t^{2}\left(\operatorname{rot}\left(\boldsymbol{\alpha}_{h}-\widetilde{\boldsymbol{\alpha}_{h}}\right), q\right)=0 \quad \forall q \in{\stackrel{\circ}{Q_{h}}}_{(}\left(G_{0}^{\prime}\right), \\
\left(\boldsymbol{\alpha}_{h}-\widetilde{\boldsymbol{\alpha}_{h}}, \boldsymbol{\delta}\right)-\left(p_{h}-\widetilde{p_{h}}, \operatorname{rot} \delta\right)=0 \quad \forall \boldsymbol{\delta} \in \stackrel{\circ}{\Gamma}_{h}\left(G_{0}^{\prime}\right) .
\end{gathered}
$$

We split the error functions on $G_{0}$ as follows:

$$
\begin{aligned}
\boldsymbol{\theta}-\boldsymbol{\theta}_{h} & =\boldsymbol{\theta}-\tilde{\boldsymbol{\theta}}_{h}+\tilde{\boldsymbol{\theta}}_{h}-\boldsymbol{\theta}_{h}, \\
p-p_{h} & =p-\widetilde{p_{h}}+\widetilde{p_{h}}-p_{h}, \\
\boldsymbol{\alpha}-\boldsymbol{\alpha}_{h} & =\boldsymbol{\alpha}-\widetilde{\boldsymbol{\alpha}_{h}}+\widetilde{\boldsymbol{\alpha}_{h}}-\boldsymbol{\alpha}_{h} .
\end{aligned}
$$

The estimate (6.2) is now a straightforward consequence of the two propositions 
below. In fact the triangle inequality, (6.3) and Proposition 6.4 give

$$
\begin{aligned}
\|(\boldsymbol{\theta}- & \left.\boldsymbol{\theta}_{h}, \boldsymbol{\alpha}-\boldsymbol{\alpha}_{h}, p-p_{h}\right) \|_{1}, G_{0} \\
\leq & \left\|\left(\tilde{\boldsymbol{\theta}}-\tilde{\boldsymbol{\theta}}_{h}, \tilde{\boldsymbol{\alpha}}-\widetilde{\boldsymbol{\alpha}_{h}}, \tilde{p}-\widetilde{p_{h}}\right)\right\|_{1, G} \\
& +\left\|\left(\tilde{\boldsymbol{\theta}}_{h}-\boldsymbol{\theta}_{h}, \widetilde{\boldsymbol{\alpha}_{h}}-\boldsymbol{\alpha}_{h}, \widetilde{p_{h}}-p_{h}\right)\right\|_{1, G_{0}} \\
\leq & \left\|\left(\tilde{\boldsymbol{\theta}}-\tilde{\boldsymbol{\theta}}_{h}, \tilde{\boldsymbol{\alpha}}-\widetilde{\boldsymbol{\alpha}_{h}}, \tilde{p}-\widetilde{p_{h}}\right)\right\|_{1, G} \\
& +C\left\|\omega \psi-P_{1}(\omega \psi)\right\|_{1, G_{0}^{\prime}}+C\left\|\psi-\psi_{h}\right\|_{1, G_{0}^{\prime}} \\
& +C\left\|\left(\tilde{\boldsymbol{\theta}}-\tilde{\boldsymbol{\theta}}_{h}, \tilde{\boldsymbol{\alpha}}-\widetilde{\boldsymbol{\alpha}_{h}}, \tilde{p}-\widetilde{p_{h}}\right)\right\|_{-\mu_{2}, G_{0}^{\prime}} \\
& +C\left\|\left(\boldsymbol{\theta}-\boldsymbol{\theta}_{h}, \boldsymbol{\alpha}-\boldsymbol{\alpha}_{h}, p-p_{h}\right)\right\|_{-\mu_{2}, G_{0}^{\prime}} \\
\leq & (1+C)\left\|\left(\tilde{\boldsymbol{\theta}}-\tilde{\boldsymbol{\theta}}_{h}, \tilde{\boldsymbol{\alpha}}-\widetilde{\boldsymbol{\alpha}_{h}}, \tilde{p}-\widetilde{p_{h}}\right)\right\|_{1, G} \\
& +C\left\|\left(\boldsymbol{\theta}-\boldsymbol{\theta}_{h}, \boldsymbol{\alpha}-\boldsymbol{\alpha}_{h}, p-p_{h}\right)\right\|-\mu_{2}, G_{0}^{\prime} \\
& +C\left\|\omega \psi-P_{1}(\omega \psi)\right\|_{1, G_{0}^{\prime}}+C\left\|\psi-\psi_{h}\right\|_{1, G_{0}^{\prime}} .
\end{aligned}
$$

Then we obtain (6.2) by applying Proposition 6.3.

Next, the approximation properties of the finite element spaces considered imply

Corollary 6.2. Under the same hypotheses as in Theorem 6.1, we have

$$
\begin{aligned}
& \left\|\left(\boldsymbol{\theta}-\boldsymbol{\theta}_{h}, \boldsymbol{\alpha}-\boldsymbol{\alpha}_{h}, p-p_{h}\right)\right\|_{1, G_{0}} \\
& \leq \quad C\left(\left\|\psi-\psi_{h}\right\|_{1, G}+h^{k}\|\psi\|_{k+1, G}\right)+C h^{k}\|(\boldsymbol{\theta}, \boldsymbol{\alpha}, p)\|_{k+1, G} \\
& \quad+C\left\|\left(\boldsymbol{\theta}-\boldsymbol{\theta}_{h}, \boldsymbol{\alpha}-\boldsymbol{\alpha}_{h}, p-p_{h}\right)\right\|-\mu_{2}, G,
\end{aligned}
$$

where $\mu_{2}$ is a nonnegative integer.

Proof. Applying Remark 4.1, we easily see that

$$
\left\|\left(\omega \boldsymbol{\theta}-\Pi(\omega \boldsymbol{\theta}), \omega \boldsymbol{\alpha}-\mathbf{R}(\omega \boldsymbol{\alpha}), \omega p-P_{0}(\omega p)\right)\right\|_{1, G} \leq C h^{k}\|(\boldsymbol{\theta}, \boldsymbol{\alpha}, p)\|_{k+1, G}
$$

and

$$
\left\|\omega \psi-P_{1}(\omega \psi)\right\|_{1, G} \leq C h^{k}\|\psi\|_{k+1, G} .
$$

On the other hand, by (3.21), we have that for every $\boldsymbol{\eta} \in \stackrel{\circ}{\boldsymbol{\Theta}}_{h}(G)$ there holds

$$
(\operatorname{rot}(\boldsymbol{\eta}-\mathbf{R} \boldsymbol{\eta}), q)=0 \quad \forall q \in Q_{h} .
$$

Integrating by parts, we get

$$
(\boldsymbol{\eta}-\mathbf{R} \boldsymbol{\eta}, \operatorname{rot} q)=0 \quad \forall q \in Q_{h} .
$$

This means that $\boldsymbol{\eta}-\mathbf{R} \boldsymbol{\eta}$ is orthogonal to the set of piecewise polynomials of degree less than or equal to $k-2$. Let $\operatorname{grad}(\omega \psi)^{*}$ be the interpolant of $\operatorname{grad}(\omega \psi)$ in the set of piecewise polynomials of degree $k-2$; then

$$
\begin{aligned}
\sup _{\substack{\dot{\boldsymbol{\eta}} \in \dot{\Theta}_{h}(G)\\
}} \frac{(\operatorname{grad}(\omega \psi), \boldsymbol{\eta}-\mathbf{R} \boldsymbol{\eta})}{\|\boldsymbol{\eta}\|_{1, G}} & =\sup _{\substack{\circ \\
\boldsymbol{\eta} \in \dot{\Theta}_{h}(G)}} \frac{\left(\operatorname{grad}(\omega \psi)-\operatorname{grad}(\omega \psi)^{*}, \boldsymbol{\eta}-\mathbf{R} \boldsymbol{\eta}\right)}{\|\boldsymbol{\eta}\|_{1, G}} \\
& \leq C h^{k}\|\psi\|_{k, G} .
\end{aligned}
$$

Inserting these estimates into the right-hand side of (6.2), we get (6.20). 
Proposition 6.3. Let $\tilde{\boldsymbol{\theta}}, \tilde{p}, \tilde{\boldsymbol{\alpha}}$ be given by (6.4) and let $\tilde{\boldsymbol{\theta}}_{h}, \widetilde{p_{h}}, \widetilde{\boldsymbol{\alpha}_{h}}$ be the solution of (6.8)-(6.10). Let $\mathrm{A} 1$ hold; then

$$
\begin{aligned}
\left\|\left(\tilde{\boldsymbol{\theta}}-\tilde{\boldsymbol{\theta}}_{h}, \tilde{\boldsymbol{\alpha}}-\widetilde{\boldsymbol{\alpha}_{h}}, \tilde{p}-\widetilde{p_{h}}\right)\right\|_{1, G} & \\
\leq C & \left(\left\|\left(\tilde{\boldsymbol{\theta}}-\Pi \tilde{\boldsymbol{\theta}}, \tilde{\boldsymbol{\alpha}}-\mathbf{R} \tilde{\boldsymbol{\alpha}}, \tilde{p}-P_{0} \tilde{p}\right)\right\|_{1, G}\right. \\
& \left.+\left\|\omega \psi-P_{1}(\omega \psi)\right\|_{1, G}^{2}+\sup _{\substack{\boldsymbol{\eta} \in \dot{\boldsymbol{\theta}}_{h}(G)\\
}} \frac{(\operatorname{grad}(\omega \psi), \boldsymbol{\eta}-\mathbf{R} \boldsymbol{\eta})}{\|\boldsymbol{\eta}\|_{1, G}}\right) .
\end{aligned}
$$

Proof. The proof of this proposition is quite standard and relies on the assumption A1 and on the properties (3.19), (3.21), (3.22) of the finite element spaces (see [11] for technical details).

Proposition 6.4. Under the same assumptions as in Theorem 6.1, the following estimate holds:

$$
\begin{aligned}
\left\|\left(\tilde{\boldsymbol{\theta}}_{h}-\boldsymbol{\theta}_{h}, \widetilde{\boldsymbol{\alpha}_{h}}-\boldsymbol{\alpha}_{h}, \widetilde{p_{h}}-p_{h}\right)\right\|_{1, G_{0}} \\
\leq C\left\|\operatorname{grad}\left(P_{1}(\omega \psi)-\psi_{h}\right)\right\|_{0, G_{0}^{\prime}} \\
\quad+C\left\|\left(\tilde{\boldsymbol{\theta}}_{h}-\boldsymbol{\theta}_{h}, \widetilde{\boldsymbol{\alpha}_{h}}-\boldsymbol{\alpha}_{h}, \widetilde{p_{h}}-p_{h}\right)\right\|_{-\mu_{2}, G_{0}^{\prime}}
\end{aligned}
$$

where $\mu_{2}$ is a nonnegative integer.

The proof of this proposition will be given in $\S 7$.

We are now in a position to prove the interior estimates:

Theorem 6.5. Let $\mathrm{A} 1-\mathrm{A} 3$ hold, and let $\Omega_{0} \subset \subset \Omega_{1} \subset \subset \Omega$; then for $h$ sufficiently small we have

$$
\begin{aligned}
& \left\|\left(\boldsymbol{\theta}-\boldsymbol{\theta}_{h}, \boldsymbol{\alpha}-\boldsymbol{\alpha}_{h}, p-p_{h}\right)\right\|_{1, \Omega_{0}} \\
& \quad \leq C h^{k}\left(\|\psi\|_{k+1, \Omega_{1}}+\|(\boldsymbol{\theta}, \boldsymbol{\alpha}, p)\|_{k+1, \Omega_{1}}\right) \\
& \quad+C\left(\left\|\psi-\psi_{h}\right\|_{-\mu_{1}, \Omega_{1}}+\left\|\left(\boldsymbol{\theta}-\boldsymbol{\theta}_{h}, \boldsymbol{\alpha}-\boldsymbol{\alpha}_{h}, p-p_{h}\right)\right\|_{-\mu_{2}, \Omega_{1}}\right),
\end{aligned}
$$

where $\mu_{1}$ and $\mu_{2}$ are two nonnegative integers.

Proof. Let $\Omega_{0}^{\prime}$ be such that $\Omega_{0} \subset \subset \Omega_{0}^{\prime} \subset \subset \Omega_{1} \subset \subset \Omega$. We cover $\bar{\Omega}_{0}$ with a finite number of spheres $G_{0}\left(x_{i}\right), i=1, \ldots, n$, centered at $x_{i} \in \bar{\Omega}_{0}$, with $\operatorname{diam}\left(G_{0}\left(x_{i}\right)\right)=\frac{1}{2} \operatorname{dist}\left(\bar{\Omega}_{0}, \partial \Omega_{0}^{\prime}\right)$. Then, let $G\left(x_{i}\right), i=1, \ldots, n$, be corresponding concentric spheres with $\operatorname{diam}\left(G\left(x_{i}\right)\right)=\operatorname{dist}\left(\bar{\Omega}_{0}, \partial \Omega_{0}^{\prime}\right)$. We apply Corollary 6.2 to each pair of centered spheres and we have, for $i=1, \ldots, n$,

$$
\begin{aligned}
&\left\|\left(\boldsymbol{\theta}-\boldsymbol{\theta}_{h}, \boldsymbol{\alpha}-\boldsymbol{\alpha}_{h}, p-p_{h}\right)\right\|_{1, G_{0}\left(x_{i}\right)} \\
& \leq C_{i}\left(\left\|\psi-\psi_{h}\right\|_{1, G\left(x_{i}\right)}+h^{k}\|\psi\|_{k+1, G\left(x_{i}\right)}\right) \\
&+C\left(h^{k}\|(\boldsymbol{\theta}, \boldsymbol{\alpha}, p)\|_{k+1}, G\left(x_{i}\right)+C\left\|\left(\boldsymbol{\theta}-\boldsymbol{\theta}_{h}, \boldsymbol{\alpha}-\boldsymbol{\alpha}_{h}, p-p_{h}\right)\right\|_{-\mu_{2}, G\left(x_{i}\right)}\right) \\
& \leq C_{i}\left(\left\|\psi-\psi_{h}\right\|_{1, \Omega_{0}^{\prime}}+h^{k}\|\psi\|_{k+1, \Omega_{0}^{\prime}}\right) \\
&+C\left(h^{k}\|(\boldsymbol{\theta}, \boldsymbol{\alpha}, p)\|_{k+1, \Omega_{0}^{\prime}}+C\left\|\left(\boldsymbol{\theta}-\boldsymbol{\theta}_{h}, \boldsymbol{\alpha}-\boldsymbol{\alpha}_{h}, p-p_{h}\right)\right\|_{-\mu_{2}, \Omega_{0}^{\prime}}\right) .
\end{aligned}
$$

Therefore, (6.1), written with $\Omega_{0}^{\prime}$ instead of $\Omega_{0}$, gives (6.24).

Remark 6.6. As we have pointed out in Remark 4.2, rectangular families of finite element spaces defined by (3.43)-(3.46) do not satisfy Assumption A3. However, we can get the same results as in Theorems 6.1 and 6.5 also in this case, by assuming that $t \leq C h$ in the interior of the domain $\Omega$ (see Remark 7.10 below), which is reasonable in a good number of applications. 
6.3. Interior error estimates for the last Poisson equation. It remains to analyze the interior error estimates for the last Poisson equation (3.37) discretized by (3.32). In this case the error equation can be written as follows:

$$
\left(\operatorname{grad}\left(w-w_{h}\right), \operatorname{grad} v\right)=\left(\boldsymbol{\theta}-\mathbf{R} \boldsymbol{\theta}_{h}, \operatorname{grad} v\right) \quad \forall v \in W_{h} .
$$

Let $\Omega_{0} \subset \subset \Omega_{0}^{\prime} \subset \subset \Omega_{0}^{\prime \prime} \subset \subset \Omega_{1} \subset \subset \Omega$; we apply the results of Theorem 5.2 of [14] and get

$$
\left\|w-w_{h}\right\|_{1, \Omega_{0}} \leq C\left(h^{k}\|w\|_{k+1, \Omega_{0}^{\prime}}+\left\|w-w_{h}\right\|_{-\mu_{3}, \Omega_{0}^{\prime}}+\left\|\boldsymbol{\theta}-\mathbf{R} \boldsymbol{\theta}_{h}\right\|_{0, \Omega_{0}^{\prime}}\right)
$$

where $\mu_{3}$ is a nonnegative integer.

To bound the last term in (6.26), we use the triangle inequality and find easily that

$$
\left\|\boldsymbol{\theta}-\mathbf{R} \boldsymbol{\theta}_{h}\right\|_{0, \Omega_{0}^{\prime}} \leq\|\boldsymbol{\theta}-\mathbf{R} \boldsymbol{\theta}\|_{0, \Omega_{0}^{\prime}}+\left\|\mathbf{R}\left(\boldsymbol{\theta}-\boldsymbol{\theta}_{h}\right)\right\|_{0, \Omega_{0}^{\prime}}
$$

From $\mathrm{A} 1$ it then follows that

$$
\|\boldsymbol{\theta}-\mathbf{R} \boldsymbol{\theta}\|_{0, \Omega_{0}^{\prime}} \leq C h^{k}\|\boldsymbol{\theta}\|_{k, \Omega_{0}^{\prime \prime}}
$$

and

$$
\left\|\mathbf{R}\left(\boldsymbol{\theta}-\boldsymbol{\theta}_{h}\right)\right\|_{0, \Omega_{0}^{\prime}} \leq C\left\|\boldsymbol{\theta}-\boldsymbol{\theta}_{h}\right\|_{1, \Omega_{0}^{\prime \prime}}
$$

Using (6.24) with $\Omega_{0}^{\prime \prime}$ instead of $\Omega_{0}$, we obtain

$$
\begin{aligned}
\left\|w-w_{h}\right\|_{1, \Omega_{0}} \leq & C h^{k}\left(\|\psi\|_{k+1, \Omega_{1}}+\|w\|_{k+1, \Omega_{1}}+\|(\boldsymbol{\theta}, \boldsymbol{\alpha}, p)\|_{k+1, \Omega_{1}}\right) \\
& +C\left(\left\|\psi-\psi_{h}\right\|_{-\mu_{1}, \Omega_{1}}+\left\|\left(\boldsymbol{\theta}-\boldsymbol{\theta}_{h}, \boldsymbol{\alpha}-\boldsymbol{\alpha}_{h}, p-p_{h}\right)\right\|_{-\mu_{2}, \Omega_{1}}\right. \\
& \left.+\left\|w-w_{h}\right\|_{-\mu_{3}, \Omega_{1}}\right) .
\end{aligned}
$$

\section{LOCAL INTERIOR STABILITY AND DUALITY ESTIMATES}

In this section we shall discuss the properties of $\boldsymbol{\theta}_{h}-\tilde{\boldsymbol{\theta}}_{h}, \boldsymbol{\alpha}_{h}-\widetilde{\boldsymbol{\alpha}}_{h}, p_{h}-$ $\widetilde{p_{h}}$ which satisfy (6.14)-(6.16). Abusing notation, we replace in (6.14)-(6.16) $\boldsymbol{\theta}_{h}-\tilde{\boldsymbol{\theta}}_{h}, \boldsymbol{\alpha}_{h}-\widetilde{\boldsymbol{\alpha}_{h}}, p_{h}-\widetilde{p_{h}}$, and $\psi_{h}-P_{1}(\omega \psi)$ by $\boldsymbol{\theta}_{h} \in \boldsymbol{\Theta}_{h}\left(\Omega_{1}\right), \boldsymbol{\alpha}_{h} \in \Gamma_{h}\left(\Omega_{1}\right)$, $p_{h} \in Q_{h}\left(\Omega_{1}\right)$, and $\psi_{h} \in W_{h}\left(\Omega_{1}\right)$, so that we have the following system:

$$
\begin{gathered}
\mathbf{a}\left(\boldsymbol{\theta}_{h}, \boldsymbol{\eta}\right)-\left(p_{h}, \operatorname{rot} \boldsymbol{\eta}\right)=\left(\operatorname{grad} \psi_{h}, \mathbf{R} \boldsymbol{\eta}\right) \quad \forall \boldsymbol{\eta} \in{\stackrel{\circ}{\boldsymbol{\Theta}_{h}}}_{\left(\Omega_{1}\right)}, \\
\left(\operatorname{rot} \boldsymbol{\theta}_{h}, q\right)+\lambda^{-1} t^{2}\left(\operatorname{rot} \boldsymbol{\alpha}_{h}, q\right)=0 \quad \forall q \in \stackrel{\circ}{Q}_{h}\left(\Omega_{1}\right), \\
\left(\boldsymbol{\alpha}_{h}, \boldsymbol{\delta}\right)-\left(p_{h}, \operatorname{rot} \boldsymbol{\delta}\right)=0 \quad \forall \boldsymbol{\delta} \in \stackrel{\circ}{\Gamma}_{h}\left(\Omega_{1}\right) .
\end{gathered}
$$

Then we analyze this set of equations in order to obtain interior stability estimates for $\boldsymbol{\theta}_{h}, \boldsymbol{\alpha}_{h}$, and $p_{h}$ in terms of a negative norm of the same functions on a slightly bigger domain. To do this, we shall need some interior duality estimates for these functions.

Throughout this section we assume that A1-A3 hold.

Let $G_{0} \subset \subset G_{1} \subset \subset G_{1}^{\prime}$ be concentric spheres all contained in $\Omega_{1}$ and consider a cutoff function

$$
\omega \in \mathbf{C}_{0}^{\infty}\left(G_{1}\right), \quad \text { with } \omega \equiv 1 \text { on } G_{0}
$$


Then we have

$$
\begin{aligned}
\left\|\boldsymbol{\theta}_{h}\right\|_{1, G_{0}}^{2}+\lambda^{-1} t^{2}\left\|\boldsymbol{\alpha}_{h}\right\|_{0, G_{0}}^{2} & =\left\|\omega \boldsymbol{\theta}_{h}\right\|_{1, G_{0}}^{2}+\lambda^{-1} t^{2}\left\|\omega \boldsymbol{\alpha}_{h}\right\|_{0, G_{0}}^{2} \\
& =\left\|\omega \boldsymbol{\theta}_{h}\right\|_{1, G_{1}^{\prime}}^{2}+\lambda^{-1} t^{2}\left\|\omega \boldsymbol{\alpha}_{h}\right\|_{0, G_{1}^{\prime}}^{2}
\end{aligned}
$$

Owing to (7.4) and Remark 4.1, $\Pi\left(\omega \boldsymbol{\theta}_{h}\right) \in \stackrel{\circ}{\boldsymbol{\Theta}}_{h}\left(G_{1}^{\prime}\right)$ and $\mathbf{R}\left(\omega \boldsymbol{\alpha}_{h}\right) \in \stackrel{\circ}{\Gamma}_{h}\left(G_{1}^{\prime}\right)$ if $h$ is sufficiently small. Then the triangle inequality and (4.5), (4.6) give

$$
\begin{aligned}
\left\|\boldsymbol{\theta}_{h}\right\|_{1, G_{0}}^{2}+\lambda^{-1} t^{2}\left\|\boldsymbol{\alpha}_{h}\right\|_{0, G_{0}}^{2} \leq & C h^{2}\left(\left\|\boldsymbol{\theta}_{h}\right\|_{1, G_{1}^{\prime}}^{2}+\lambda^{-1} t^{2}\left\|\boldsymbol{\alpha}_{h}\right\|_{0, G_{1}^{\prime}}^{2}\right) \\
& +\left\|\Pi\left(\omega \boldsymbol{\theta}_{h}\right)\right\|_{1, G_{1}^{\prime}}^{2}+\lambda^{-1} t^{2}\left\|\mathbf{R}\left(\omega \boldsymbol{\alpha}_{h}\right)\right\|_{0, G_{1}^{\prime}}^{2}
\end{aligned}
$$

We estimate the last two terms of (7.5) in the next lemmas.

Lemma 7.1. For $h$ sufficiently small, there exists a constant independent of $h$ and $t$ such that

$$
\begin{aligned}
&\left\|\Pi\left(\omega \boldsymbol{\theta}_{h}\right)\right\|_{1, G_{1}^{\prime}}^{2}+\lambda^{-1} t^{2}\left\|\mathbf{R}\left(\omega \boldsymbol{\alpha}_{h}\right)\right\|_{0, G_{1}^{\prime}}^{2} \\
& \leq C h^{2}\left(\left\|\boldsymbol{\theta}_{h}\right\|_{1, G_{1}^{\prime}}^{2}+\lambda^{-1} t^{2}\left\|\boldsymbol{\alpha}_{h}\right\|_{0, G_{1}^{\prime}}^{2}+\left\|p_{h}\right\|_{0, G_{1}^{\prime}}^{2}\right) \\
&+C\left(\left\|\boldsymbol{\theta}_{h}\right\|_{0, G_{1}^{\prime}}^{2}+\left\|p_{h}\right\|_{-1, G_{1}^{\prime}}^{2}+\left\|\operatorname{grad} \psi_{h}\right\|_{0, G_{1}^{\prime}}^{2}\right) \\
&+\lambda^{-1} t^{2}\left(\omega p_{h},-\operatorname{rot} \omega \cdot \boldsymbol{\alpha}_{h}\right)+\lambda^{-1} t^{2}\left(p_{h},-\operatorname{rot} \omega \cdot \mathbf{R}\left(\omega \boldsymbol{\alpha}_{h}\right)\right) \\
&+\varepsilon_{1}\left\|\omega p_{h}\right\|_{0, G_{1}}^{2}
\end{aligned}
$$

with $\varepsilon_{1}>0$ arbitrary.

Proof. We apply assumption A2 and Lemma 4.3 with $s=-1$ and get

$$
\begin{aligned}
& \mathbf{a}\left(\Pi\left(\omega \boldsymbol{\theta}_{h}\right), \Pi\left(\omega \boldsymbol{\theta}_{h}\right)\right)+\lambda^{-1} t^{2}\left\|\mathbf{R}\left(\omega \boldsymbol{\alpha}_{h}\right)\right\|_{0, G_{0}}^{2} \\
&=\mathbf{a}\left(\Pi\left(\omega \boldsymbol{\theta}_{h}\right)-\omega \boldsymbol{\theta}_{h}, \Pi\left(\omega \boldsymbol{\theta}_{h}\right)\right)+\lambda^{-1} t^{2}\left(\mathbf{R}\left(\omega \boldsymbol{\alpha}_{h}\right)-\omega \boldsymbol{\alpha}_{h}, \mathbf{R}\left(\omega \boldsymbol{\alpha}_{h}\right)\right) \\
& \quad+\mathbf{a}\left(\omega \boldsymbol{\theta}_{h}, \Pi\left(\omega \boldsymbol{\theta}_{h}\right)\right)+\lambda^{-1} t^{2}\left(\omega \boldsymbol{\alpha}_{h}, \mathbf{R}\left(\omega \boldsymbol{\alpha}_{h}\right)\right) \\
& \leq C h\left(\left\|\boldsymbol{\theta}_{h}\right\|_{1, G_{1}^{\prime}}\left\|\Pi\left(\omega \boldsymbol{\theta}_{h}\right)\right\|_{1, G_{1}^{\prime}}+\lambda^{-1} t^{2}\left\|\boldsymbol{\alpha}_{h}\right\|_{0, G_{1}^{\prime}}\left\|\mathbf{R}\left(\omega \boldsymbol{\alpha}_{h}\right)\right\|_{0, G_{1}^{\prime}}\right) \\
&+C\left\|\boldsymbol{\theta}_{h}\right\|_{0, G_{1}^{\prime}}\left\|\Pi\left(\omega \boldsymbol{\theta}_{h}\right)\right\|_{1, G_{1}^{\prime}}+\mathbf{a}\left(\boldsymbol{\theta}_{h}, \omega \Pi\left(\omega \boldsymbol{\theta}_{h}\right)\right)+\lambda^{-1} t^{2}\left(\boldsymbol{\alpha}_{h}, \omega \mathbf{R}\left(\omega \boldsymbol{\alpha}_{h}\right)\right) .
\end{aligned}
$$

We proceed by analyzing the last two terms. We use again $\mathrm{A} 2$, the equations (7.1), and (7.3) and obtain

$$
\begin{aligned}
\mathbf{a}\left(\boldsymbol{\theta}_{h},\right. & \left.\omega \Pi\left(\omega \boldsymbol{\theta}_{h}\right)\right)+\lambda^{-1} t^{2}\left(\boldsymbol{\alpha}_{h}, \omega \mathbf{R}\left(\omega \boldsymbol{\alpha}_{h}\right)\right) \\
\leq & C h\left(\left\|\boldsymbol{\theta}_{h}\right\|_{1, G_{1}^{\prime}}\left\|\Pi\left(\omega \boldsymbol{\theta}_{h}\right)\right\|_{1, G_{1}^{\prime}}+\lambda^{-1} t^{2}\left\|\boldsymbol{\alpha}_{h}\right\|_{0, G_{1}^{\prime}}\left\|\mathbf{R}\left(\omega \boldsymbol{\alpha}_{h}\right)\right\|_{0, G_{1}^{\prime}}\right) \\
& +\left(p_{h}, \operatorname{rot} \Pi\left(\omega \Pi\left(\omega \boldsymbol{\theta}_{h}\right)\right)\right)+\left(\operatorname{grad} \psi_{h}, \mathbf{R} \Pi\left(\omega \Pi\left(\omega \boldsymbol{\theta}_{h}\right)\right)\right) \\
& +\lambda^{-1} t^{2}\left(p_{h}, \operatorname{rot} \mathbf{R}\left(\omega \mathbf{R}\left(\omega \boldsymbol{\alpha}_{h}\right)\right)\right) \\
\leq & C h\left(\left\|\boldsymbol{\theta}_{h}\right\|_{1, G_{1}^{\prime}}\left\|\Pi\left(\omega \boldsymbol{\theta}_{h}\right)\right\|_{1, G_{1}^{\prime}}+\lambda^{-1} t^{2}\left\|\boldsymbol{\alpha}_{h}\right\|_{0, G_{1}^{\prime}}\left\|\mathbf{R}\left(\omega \boldsymbol{\alpha}_{h}\right)\right\|_{0, G_{1}^{\prime}}\right) \\
& +C\left\|\operatorname{grad} \psi_{h}\right\|_{0, G_{1}^{\prime}}\left\|\Pi\left(\omega \boldsymbol{\theta}_{h}\right)\right\|_{1, G_{1}^{\prime}}+T_{1}+\lambda^{-1} t^{2} T_{2} .
\end{aligned}
$$

We apply twice (4.14) to $T_{1}$ and we get

$$
\begin{aligned}
T_{1}= & \left(p_{h}, \operatorname{rot} \Pi\left(\omega \Pi\left(\omega \boldsymbol{\theta}_{h}\right)\right)\right) \\
= & \left(p_{h},-\operatorname{rot} \omega \cdot \Pi\left(\omega \boldsymbol{\theta}_{h}\right)\right)+\left(\omega p_{h}-P_{0}\left(\omega p_{h}\right), \operatorname{rot} \Pi\left(\omega \boldsymbol{\theta}_{h}\right)\right) \\
& +\left(P_{0}\left(\omega p_{h}\right), \operatorname{rot} \Pi\left(\omega \boldsymbol{\theta}_{h}\right)\right) \\
= & \left(p_{h},-\operatorname{rot} \omega \cdot \Pi\left(\omega \boldsymbol{\theta}_{h}\right)\right)+\left(\omega p_{h}-P_{0}\left(\omega p_{h}\right), \operatorname{rot} \Pi\left(\omega \boldsymbol{\theta}_{h}\right)\right) \\
& +\left(P_{0}\left(\omega p_{h}\right),-\operatorname{rot} \omega \cdot \boldsymbol{\theta}_{h}\right)+\left(\omega P_{0}\left(\omega p_{h}\right)-P_{0}\left(\omega P_{0}\left(\omega p_{h}\right)\right), \operatorname{rot} \theta_{h}\right) \\
& +\left(P_{0}\left(\omega P_{0}\left(\omega p_{h}\right)\right), \operatorname{rot} \theta_{h}\right) .
\end{aligned}
$$


To evaluate $T_{2}$, we use first (4.15) and then (3.19); hence we obtain

$$
\begin{aligned}
T_{2} & =\left(p_{h}, \operatorname{rot} \mathbf{R}\left(\omega \mathbf{R}\left(\omega \boldsymbol{\alpha}_{h}\right)\right)\right) \\
& =\left(p_{h},-\operatorname{rot} \omega \cdot \mathbf{R}\left(\omega \boldsymbol{\alpha}_{h}\right)\right)+\left(\omega p_{h}, \operatorname{rot} \mathbf{R}\left(\omega \boldsymbol{\alpha}_{h}\right)\right) .
\end{aligned}
$$

The last term in the right-hand side can be written as follows, thanks to the commuting diagram property (3.19):

(7.10)

$$
\begin{aligned}
\left(\omega p_{h}, \operatorname{rot} \mathbf{R}\left(\omega \boldsymbol{\alpha}_{h}\right)\right)= & \left(\omega p_{h}, \operatorname{rot} \mathbf{R}\left(\omega \boldsymbol{\alpha}_{h}\right)-\operatorname{rot}\left(\omega \boldsymbol{\alpha}_{h}\right)\right) \\
& +\left(\omega p_{h},-\operatorname{rot} \omega \cdot \boldsymbol{\alpha}_{h}\right)+\left(\omega p_{h}, \omega \operatorname{rot} \boldsymbol{\alpha}_{h}\right) \\
= & \left(\omega p_{h}, \operatorname{rot} \mathbf{R}\left(\omega \boldsymbol{\alpha}_{h}\right)-\operatorname{rot}\left(\omega \boldsymbol{\alpha}_{h}\right)\right)+\left(\omega p_{h},-\operatorname{rot} \omega \cdot \boldsymbol{\alpha}_{h}\right) \\
& +\left(\omega p_{h}, \omega \operatorname{rot} \boldsymbol{\alpha}_{h}-P_{0}\left(\omega \operatorname{rot} \boldsymbol{\alpha}_{h}\right)\right) \\
& +\left(P_{0}\left(\omega P_{0}\left(\omega p_{h}\right)\right), \operatorname{rot} \boldsymbol{\alpha}_{h}\right) .
\end{aligned}
$$

Putting together (7.8)-(7.10) and using (7.2), (4.7), (4.9) and the CauchySchwarz inequality yields

(7.11)

$$
\begin{aligned}
T_{1}+\lambda^{-1} t^{2} T_{2} \leq C & {\left[\left(\left\|p_{h}\right\|_{-1, G_{1}}\left\|\Pi\left(\omega \boldsymbol{\theta}_{h}\right)\right\|_{1, G_{1}^{\prime}}+h\left\|p_{h}\right\|_{0, G_{1}^{\prime}}\left\|\Pi\left(\omega \boldsymbol{\theta}_{h}\right)\right\|_{1, G_{1}^{\prime}}\right)\right.} \\
& +\left(h\left\|p_{h}\right\|_{0, G_{1}^{\prime}}+\left\|\omega p_{h}\right\|_{0, G_{1}}\right)\left(\left\|\boldsymbol{\theta}_{h}\right\|_{0, G_{1}}+h\left\|\boldsymbol{\theta}_{h}\right\|_{1, G_{1}^{\prime}}\right) \\
& \left.+h \lambda^{-1} t^{2}\left\|\omega p_{h}\right\|_{0, G_{1}}\left(\left\|\boldsymbol{\alpha}_{h}\right\|_{0, G_{1}^{\prime}}+\left\|\operatorname{rot} \boldsymbol{\alpha}_{h}\right\|_{0, G_{h}}\right)\right] \\
+ & \lambda^{-1} t^{2}\left(p_{h},-\operatorname{rot} \omega \cdot \mathbf{R}\left(\omega \boldsymbol{\alpha}_{h}\right)\right)+\lambda^{-1} t^{2}\left(\omega p_{h},-\operatorname{rot} \omega \cdot \boldsymbol{\alpha}_{h}\right) .
\end{aligned}
$$

Now recall that (7.2) states that $\lambda^{-1} t^{2} \operatorname{rot} \alpha_{h}=-P_{0}\left(\operatorname{rot} \theta_{h}\right)$ on each element; hence,

$$
\lambda^{-1} t^{2}\left\|\operatorname{rot} \alpha_{h}\right\|_{0, G_{h}}=\left\|P_{0}\left(\operatorname{rot} \theta_{h}\right)\right\|_{0, G_{h}} \leq\left\|\theta_{h}\right\|_{1, G_{1}^{\prime}} .
$$

We insert (7.12) in (7.11), next (7.11) in (7.7), recall Korn's inequality (3.4) and obtain

$$
\begin{aligned}
& C\left\|\Pi\left(\omega \boldsymbol{\theta}_{h}\right)\right\|_{1, G_{1}^{\prime}}^{2}+\lambda^{-1} t^{2}\left\|\mathbf{R}\left(\omega \boldsymbol{\alpha}_{h}\right)\right\|_{0, G_{1}^{\prime}}^{2} \\
& \leq \varepsilon_{0}\left\|\Pi\left(\omega \boldsymbol{\theta}_{h}\right)\right\|_{1, G_{1}^{\prime}}^{2}+\varepsilon_{0} \lambda^{-1} t^{2}\left\|\mathbf{R}\left(\omega \boldsymbol{\alpha}_{h}\right)\right\|_{0, G_{1}^{\prime}}^{2} \\
&+C h^{2}\left(\left\|\boldsymbol{\theta}_{h}\right\|_{1, G_{1}^{\prime}}^{2}+\lambda^{-1} t^{2}\left\|\boldsymbol{\alpha}_{h}\right\|_{0, G_{1}^{\prime}}^{2}+\left\|p_{h}\right\|_{0, G_{1}^{\prime}}^{2}\right) \\
&+C\left(\left\|\boldsymbol{\theta}_{h}\right\|_{0, G_{1}}^{2}+\left\|p_{h}\right\|_{-1, G_{1}}^{2}+\left\|\mathbf{g r a d} \psi_{h}\right\|_{0, G_{1}^{\prime}}^{2}\right)+\varepsilon_{1}\left\|\omega p_{h}\right\|_{0, G_{1}}^{2} \\
&+\lambda^{-1} t^{2}\left(\omega p_{h},-\operatorname{rot} \omega \cdot \boldsymbol{\alpha}_{h}\right)+\lambda^{-1} t^{2}\left(p_{h},-\operatorname{rot} \omega \cdot \mathbf{R}\left(\omega \boldsymbol{\alpha}_{h}\right)\right),
\end{aligned}
$$

whence (7.6) follows by choosing $\varepsilon_{0}$ properly.

We need to bound the last three terms in (7.6).

Lemma 7.2. Let $\varepsilon_{2}>0$ be a real number; then

$$
\begin{aligned}
& \lambda^{-1} t^{2}\left(\omega p_{h},-\operatorname{rot} \omega \cdot \boldsymbol{\alpha}_{h}\right)+\lambda^{-1} t^{2}\left(p_{h},-\operatorname{rot} \omega \cdot \mathbf{R}\left(\omega \boldsymbol{\alpha}_{h}\right)\right) \\
& \leq \varepsilon_{2} \lambda^{-1} t^{2}\left\|\mathbf{R}\left(\omega \boldsymbol{\alpha}_{h}\right)\right\|_{0, G_{1}^{\prime}}^{2}+C\left\|\operatorname{grad} \psi_{h}\right\|_{0, G_{1}^{\prime}}^{2} \\
&+C h^{2}\left(\left\|\boldsymbol{\theta}_{h}\right\|_{1, G_{1}^{\prime}}^{2}+\lambda^{-1} t^{2}\left\|\boldsymbol{\alpha}_{h}\right\|_{0, G_{1}^{\prime}}^{2}\right) \\
&+C\left(\left\|\boldsymbol{\theta}_{h}\right\|_{0, G_{1}}^{2}+\lambda^{-1} t^{2}\left\|\boldsymbol{\alpha}_{h}\right\|_{-1, G_{1}}^{2}+\left\|p_{h}\right\|_{-1, G_{1}}^{2}\right) .
\end{aligned}
$$


Proof. The estimate will be obtained by a duality argument. Consider $\phi \epsilon$ $\mathbf{C}_{0}^{\infty}\left(G_{1}\right)$ such that $\int_{G_{1}} \phi d x d y=1$; then we can write

$$
\begin{aligned}
\lambda^{-1} t^{2}\left(\omega p_{h}, \operatorname{rot} \omega \cdot \boldsymbol{\alpha}_{h}\right) & =\lambda^{-1} t^{2}\left(\omega_{y} p_{h}, \omega \alpha_{h}^{1}\right)-\lambda^{-1} t^{2}\left(\omega_{x} p_{h}, \omega \alpha_{h}^{2}\right), \\
\lambda^{-1} t^{2}\left(p_{h}, \operatorname{rot} \omega \cdot \mathbf{R}\left(\omega \boldsymbol{\alpha}_{h}\right)\right) & =\lambda^{-1} t^{2}\left(\omega_{y} p_{h}, \mathbf{R}\left(\omega \boldsymbol{\alpha}_{h}\right)^{1}\right)-\lambda^{-1} t^{2}\left(\omega_{x} p_{h}, \mathbf{R}\left(\omega \boldsymbol{\alpha}_{h}\right)^{2}\right) .
\end{aligned}
$$

Let $\bar{\omega}$ be either $\omega_{x}$ or $\omega_{y}$; then setting

we have

$$
\begin{array}{ll}
\gamma_{1, i}=\left(\omega, \alpha_{h}^{i}\right), & g_{1, i}=\omega \alpha_{h}^{i}-\gamma_{1, i} \phi, \\
\gamma_{2, i}=\int_{G_{1}} \mathbf{R}\left(\omega \boldsymbol{\alpha}_{h}\right)^{i} d x d y, & g_{2, i}=\mathbf{R}\left(\omega \boldsymbol{\alpha}_{h}\right)^{i}-\gamma_{2, i} \phi,
\end{array}
$$

$$
\begin{aligned}
\lambda^{-1} t^{2}\left(\bar{\omega} p_{h}, \omega \alpha_{h}^{i}\right) & =\lambda^{-1} t^{2}\left(\bar{\omega} p_{h}, g_{1, i}\right)+\lambda^{-1} t^{2}\left(\bar{\omega} p_{h}, \gamma_{1, i} \phi\right), \\
\lambda^{-1} t^{2}\left(\bar{\omega} p_{h}, \mathbf{R}\left(\omega \boldsymbol{\alpha}_{h}\right)^{i}\right) & =\lambda^{-1} t^{2}\left(\bar{\omega} p_{h}, g_{2, i}\right)+\lambda^{-1} t^{2}\left(\bar{\omega} p_{h}, \gamma_{2, i} \phi\right) .
\end{aligned}
$$

We observe that $g_{j, i}$ for $j, i=1,2$ belong to $\mathbf{L}^{2}\left(G_{1}\right)$ and have zero mean value; hence we can consider the solution of problem (5.1)-(5.3) on the domain $G_{1}$ corresponding to the data $\mathbf{f}=\phi=0$ and $g=\lambda^{-1} t^{2} g_{j, i}$ for $j, i=1,2$. Integration by parts then gives

$$
\begin{aligned}
\lambda^{-1} t^{2}\left(\bar{\omega} p_{h}, g_{j, i}\right)= & -\left(\bar{\omega} p_{h}, \operatorname{rot} \mathbf{z}\right)-\lambda^{-1} t^{2}\left(\bar{\omega} p_{h}, \operatorname{rot} \operatorname{rot} r\right) \\
& +\mathbf{a}\left(\bar{\omega} \boldsymbol{\theta}_{h}, \mathbf{z}\right)-\left(\operatorname{rot}\left(\bar{\omega} \boldsymbol{\theta}_{h}\right), r\right) .
\end{aligned}
$$

After some calculations using Lemmas 4.3 and 4.4, properties (3.19) and (3.21), and equations (7.1)-(7.3), we arrive at

$$
\begin{aligned}
\lambda^{-1} t^{2}\left(\bar{\omega} p_{h}, g_{j, i}\right)= & \mathbf{a}\left(\bar{\omega} \mathbf{z}-\Pi(\bar{\omega} \mathbf{z}), \boldsymbol{\theta}_{h}\right)-\lambda^{-1} t^{2}\left(\boldsymbol{\alpha}_{h}, \mathbf{R}(\bar{\omega} \operatorname{rot} r)-\bar{\omega} \operatorname{rot} r\right) \\
& -\left(\bar{\omega} r-P_{0}(\bar{\omega} r), \operatorname{rot} \boldsymbol{\theta}_{h}\right) \\
& +\left(\operatorname{grad} \psi_{h}, \mathbf{R} \Pi(\bar{\omega} \mathbf{z})\right)-\left(\operatorname{rot} \bar{\omega} \cdot \mathbf{z}, p_{h}\right) \\
& -\lambda^{-1} t^{2}\left(\operatorname{rot} \bar{\omega} \cdot \operatorname{rot} r, p_{h}\right)-I\left(\bar{\omega} \boldsymbol{\theta}_{h}, \mathbf{z}\right) \\
& -\lambda^{-1} t^{2}\left((\operatorname{rot} \bar{\omega}) r, \boldsymbol{\alpha}_{h}\right)+\left(r, \operatorname{rot} \bar{\omega} \cdot \boldsymbol{\theta}_{h}\right) .
\end{aligned}
$$

Then we bound all the terms using A1, (4.13), and duality estimates; one obtains

$$
\begin{aligned}
& \lambda^{-1} t^{2}\left(\bar{\omega} p_{h}, g_{j, i}\right) \leq C\left[h\left\|\boldsymbol{\theta}_{h}\right\|_{1, G_{1}^{\prime}}\left(\|\mathbf{z}\|_{2, G_{1}}+\|r\|_{1, G_{1}}\right)+\lambda^{-1} t^{2} h\left\|\boldsymbol{\alpha}_{h}\right\|_{0, G_{1}^{\prime}}\|r\|_{2, G_{1}}\right. \\
& +\left\|\operatorname{grad} \psi_{h}\right\|_{0, G_{1}^{\prime}}\|\mathbf{z}\|_{1, G_{1}} \\
& +\left\|p_{h}\right\|_{-1, G_{1}}\left(\|\mathbf{z}\|_{1, G_{1}}+\lambda^{-1} t^{2}\|r\|_{2, G_{1}}\right) \\
& \left.+\lambda^{-1} t^{2}\left\|\boldsymbol{\alpha}_{h}\right\|_{-1, G_{1}}\|r\|_{1, G_{1}}+\|r\|_{0, G_{1}}\left\|\boldsymbol{\theta}_{h}\right\|_{0, G_{1}}\right] \text {. }
\end{aligned}
$$

From the a priori estimates (5.9) we have

$$
\|\mathbf{z}\|_{1, G_{1}}+\|r\|_{0, G_{1}}+\lambda^{-1 / 2} t\|r\|_{1, G_{1}}+\lambda^{-1} t^{2}\|r\|_{2, G_{1}} \leq C \lambda^{-1} t^{2}\left\|g_{j, i}\right\|_{0, G_{1}} .
$$

Moreover, since $\mathbf{A}$ is an elliptic operator of second order with constant coefficients, we obtain from (5.1)

Hence, we conclude that

$$
\|\mathbf{z}\|_{2, G_{1}} \leq C\|\mathbf{A z}\|_{0, G_{1}} \leq C\|r\|_{1, G_{1}} \leq C \lambda^{-1 / 2} t\left\|g_{j, i}\right\|_{0, G_{1}} .
$$

$$
\begin{aligned}
& \lambda^{-1} t^{2}\left(\bar{\omega} p_{h}, g_{j, i}\right) \\
& \leq C \lambda^{-1 / 2} t\left\|g_{j, i}\right\|_{0, G_{1}}\left(h\left\|\boldsymbol{\theta}_{h}\right\|_{1, G_{1}^{\prime}}+h \lambda^{-1 / 2} t\left\|\boldsymbol{\alpha}_{h}\right\|_{0, G_{1}^{\prime}}\right. \\
& +\lambda^{-1 / 2} t\left\|\operatorname{grad} \psi_{h}\right\|_{0, G_{1}^{\prime}}+\left\|\boldsymbol{\theta}_{h}\right\|_{0, G_{1}} \\
& \left.+\lambda^{-1} t^{2}\left\|\boldsymbol{\alpha}_{h}\right\|_{-1, G_{1}}+\left\|p_{h}\right\|_{-1, G_{1}}\right) \text {. }
\end{aligned}
$$


Furthermore, we have

$$
\lambda^{-1} t^{2}\left(\bar{\omega} p_{h}, \gamma_{j, i} \phi\right) \leq C \lambda^{-1} t^{2}\left|\gamma_{j, i}\right|\left\|p_{h}\right\|_{-1, G_{1}}\|\phi\|_{1, G_{1}} .
$$

It remains only to bound $\left|\gamma_{j, i}\right|$ and $\left\|g_{j, i}\right\|_{0, G_{1}}$ for $j, i=1,2$.

Since $\omega \in \mathbf{C}_{0}^{\infty}\left(G_{1}\right)$, we have

$$
\left|\gamma_{1, i}\right|=\left|\left(\omega, \alpha_{h}^{i}\right)\right| \leq C\left\|\alpha_{h}^{i}\right\|_{-1, G_{1}} \leq C\left\|\alpha_{h}\right\|_{-1, G_{1}}
$$

and

$$
\left|\gamma_{2, i}\right| \leq\left|\int_{G_{1}} \mathbf{R}\left(\omega \boldsymbol{\alpha}_{h}\right)^{i} d x d y\right| \leq C\left\|\mathbf{R}\left(\omega \boldsymbol{\alpha}_{h}\right)^{i}\right\|_{0, G_{1}^{\prime}} \leq C\left\|\mathbf{R}\left(\omega \boldsymbol{\alpha}_{h}\right)\right\|_{0, G_{1}^{\prime}}
$$

Then

$$
\begin{aligned}
\left\|g_{1, i}\right\|_{0, G_{1}} & \leq C\left\|\omega \boldsymbol{\alpha}_{h}^{i}\right\|_{0, G_{1}}+C\left|\gamma_{1, i}\right|\|\phi\|_{0, G_{1}} \leq C\left\|\omega \boldsymbol{\alpha}_{h}\right\|_{0, G_{1}}+C\left|\gamma_{1, i}\right|\|\phi\|_{0, G_{1}} \\
& \leq C\left(h\left\|\boldsymbol{\alpha}_{h}\right\|_{0, G_{1}^{\prime}}+\left\|\mathbf{R}\left(\omega \boldsymbol{\alpha}_{h}\right)\right\|_{0, G_{1}^{\prime}}+\left\|\boldsymbol{\alpha}_{h}\right\|_{-1, G_{1}}\right),
\end{aligned}
$$

and

$$
\left\|g_{2, i}\right\|_{0, G_{1}} \leq C\left\|\mathbf{R}\left(\omega \boldsymbol{\alpha}_{h}\right)^{i}\right\|_{0, G_{1}^{\prime}}+C\left|\gamma_{2, i}\right|\|\phi\|_{0, G_{1}} \leq C\left\|\mathbf{R}\left(\omega \boldsymbol{\alpha}_{h}\right)\right\|_{0, G_{1}^{\prime}}
$$

Putting these estimates in (7.14) and (7.15), we obtain the desired inequality (7.13).

Lemma 7.3. The following estimate holds:

$$
\begin{aligned}
\left\|\omega p_{h}\right\|_{0, G_{1}} \leq & C\left\|\Pi\left(\omega \boldsymbol{\theta}_{h}\right)\right\|_{1, G_{1}^{\prime}}+C h\left(\left\|\boldsymbol{\theta}_{h}\right\|_{1, G_{1}^{\prime}}+\left\|p_{h}\right\|_{0, G_{1}^{\prime}}\right) \\
& +C\left\|p_{h}\right\|_{-1, G_{1}}+C\left\|\operatorname{grad} \psi_{h}\right\|_{0, G_{1}^{\prime}} .
\end{aligned}
$$

Proof. Let $\mathscr{A}=\left(1 / \operatorname{meas}\left(G_{1}\right)\right) \int_{G_{1}} \omega p_{h} d x d y$; then $|\mathscr{A}| \leq C\left\|p_{h}\right\|_{-1, G_{1}}$, since $\omega \in \mathbf{C}_{0}^{\infty}\left(G_{1}\right)$. Then we have $\left\|\omega p_{h}\right\|_{0, G_{1}} \leq\left\|\omega p_{h}-\mathscr{A}\right\|_{0, G_{1}}+C\left\|p_{h}\right\|_{-1, G_{1}}$, and we apply the continuous inf-sup condition as follows:

$$
\begin{aligned}
& \left\|\omega p_{h}-\mathscr{A}\right\|_{0, G_{1}} \leq C \sup _{\substack{\boldsymbol{\nu} \in\left[\dot{\mathrm{H}}^{1}\left(G_{1}\right)\right]^{2}}} \frac{\left(\omega p_{h}-\mathscr{A}, \operatorname{rot} \boldsymbol{\eta}\right)}{\|\boldsymbol{\eta}\|_{1}, G_{1}} \\
& =C \sup _{\substack{\boldsymbol{\eta} \in\left[\dot{\mathrm{H}}^{1}\left(G_{1}\right)\right]^{2}}} \frac{\left(\omega p_{h}, \operatorname{rot} \boldsymbol{\eta}\right)}{\|\boldsymbol{\eta}\|_{1, G_{1}}} \\
& \leq C h\left\|p_{h}\right\|_{0, G_{1}^{\prime}}+C \sup _{\substack{\left.\stackrel{\circ}{\dot{H}^{1}}\left(G_{1}\right)\right]^{2}}} \frac{\left(P_{0}\left(\omega p_{h}\right), \operatorname{rot} \boldsymbol{\eta}\right)}{\|\boldsymbol{\eta}\|_{1, G_{1}}} .
\end{aligned}
$$

From (3.21) and (4.14) we have for the numerator

$$
\begin{aligned}
\left(P_{0}\left(\omega p_{h}\right), \operatorname{rot} \boldsymbol{\eta}\right)= & \left(P_{0}\left(\omega p_{h}\right), \operatorname{rot} \Pi \boldsymbol{\eta}\right) \\
= & \left(p_{h}, \operatorname{rot} \Pi(\omega \Pi \boldsymbol{\eta})\right)+\left(p_{h}, \operatorname{rot} \omega \cdot \Pi \boldsymbol{\eta}\right) \\
& +\left(P_{0}\left(\omega p_{h}\right)-\omega p_{h}, \operatorname{rot} \Pi \boldsymbol{\eta}\right) .
\end{aligned}
$$

Therefore, (7.1) and Lemma 4.3 yield

$$
\begin{aligned}
\left(P_{0}\left(\omega p_{h}\right), \operatorname{rot} \boldsymbol{\eta}\right)= & \mathbf{a}\left(\boldsymbol{\theta}_{h}, \Pi(\omega \Pi \boldsymbol{\eta})-\omega \Pi \boldsymbol{\eta}\right)-\left(\operatorname{grad} \psi_{h}, \mathbf{R} \Pi(\omega \Pi \boldsymbol{\eta})\right) \\
& +\left(p_{h}, \operatorname{rot} \omega \cdot \Pi \boldsymbol{\eta}\right)+\left(P_{0}\left(\omega p_{h}\right)-\omega p_{h}, \operatorname{rot} \Pi \boldsymbol{\eta}\right) \\
& +\mathbf{a}\left(\Pi\left(\omega \boldsymbol{\theta}_{h}\right), \Pi \boldsymbol{\eta}\right)+I\left(\omega \boldsymbol{\theta}_{h}, \Pi \boldsymbol{\eta}\right)+\mathbf{a}\left(\omega \boldsymbol{\theta}_{h}-\Pi\left(\omega \boldsymbol{\theta}_{h}\right), \Pi \boldsymbol{\eta}\right) .
\end{aligned}
$$


We insert this equality in (7.17) and use (4.5), (4.7), and (4.13). Then, recalling also that by (3.22) $\|\Pi \boldsymbol{\eta}\|_{1, G_{1}^{\prime}} \leq C\|\boldsymbol{\eta}\|_{1, G_{1}}$ for every $\boldsymbol{\eta} \in\left[\stackrel{\circ}{\mathbf{H}}^{1}\left(G_{1}\right)\right]^{2}$ and that by (3.23) $\|\mathbf{R} v\|_{0, G_{1}^{\prime}} \leq C\|v\|_{1, G_{1}}$ for every $v \in \stackrel{\circ}{\boldsymbol{\Theta}}_{h}\left(G_{1}^{\prime}\right)$, we obtain (7.16).

We collect the results of the previous lemmas and we get:

Proposition 7.4. Let $G_{0} \subset \subset G_{1} \subset \subset G_{1}^{\prime}$ be concentric spheres; then there exists a constant independent of $h$ and $t$ such that

$$
\begin{aligned}
\left\|\left(\boldsymbol{\theta}_{h}, \boldsymbol{\alpha}_{h}, p_{h}\right)\right\|_{1, G_{0}} \leq & C\left(h\left\|\left(\boldsymbol{\theta}_{h}, \boldsymbol{\alpha}_{h}, p_{h}\right)\right\|_{1, G_{1}^{\prime}}+\left\|\left(\boldsymbol{\theta}_{h}, \boldsymbol{\alpha}_{h}, p_{h}\right)\right\|_{0, G_{1}}\right) \\
& +C\left\|\operatorname{grad} \psi_{h}\right\|_{0, G_{1}^{\prime}} .
\end{aligned}
$$

Proof. We put together (7.6), (7.13), and (7.16) and choose $\varepsilon_{1}$ and $\varepsilon_{2}$ properly to obtain

$$
\begin{aligned}
&\left\|\Pi\left(\omega \boldsymbol{\theta}_{h}\right)\right\|_{1, G_{1}^{\prime}}^{2}+\lambda^{-1} t^{2}\left\|\mathbf{R}\left(\omega \boldsymbol{\alpha}_{h}\right)\right\|_{0, G_{1}^{\prime}}^{2} \\
& \leq C h^{2}\left(\left\|\boldsymbol{\theta}_{h}\right\|_{1, G_{1}^{\prime}}^{2}+\lambda^{-1} t^{2}\left\|\boldsymbol{\alpha}_{h}\right\|_{0, G_{1}^{\prime}}^{2}+\left\|p_{h}\right\|_{0, G_{1}^{\prime}}^{2}\right) \\
&+C\left(\left\|\boldsymbol{\theta}_{h}\right\|_{0, G_{1}}^{2}+\lambda^{-1} t^{2}\left\|\boldsymbol{\alpha}_{h}\right\|_{-1, G_{1}}^{2}+\left\|p_{h}\right\|_{-1, G_{1}}^{2}+\left\|\operatorname{grad} \psi_{h}\right\|_{0, G_{1}^{\prime}}^{2}\right) .
\end{aligned}
$$

Hence, the estimates regarding $\theta_{h}$ and $\alpha_{h}$ in (7.18) are obtained; it remains to bound $\left\|p_{h}\right\|_{0, G_{0}}$. Using (7.4), we have $\left\|p_{h}\right\|_{0, G_{0}} \leq\left\|\omega p_{h}\right\|_{0, G_{1}}$. Then from Proposition 7.3 and (7.19) we get also the bound for $p_{h}$, and the proof is complete.

We introduce here some interior duality estimates which will be used later on to bound the second term in the right-hand side of (7.18). We give only the idea of the proofs and refer to [11] for the details.

From now on we assume that $\widehat{G}$ and $\widehat{G}_{1}$ are concentric spheres with $\widehat{G} \subset \subset$ $\widehat{G}_{1} \subset \subset \Omega_{1}$.

Lemma 7.5. Let $\widehat{G}$ and $\widehat{G}_{1}$ be as above and let $s \geq 0$ be an integer. Then for $\rho=\min (s+1, k)$ we have

$$
\begin{aligned}
\left\|\boldsymbol{\theta}_{h}\right\|_{-s, \widehat{G}} \leq & C\left\|\left(\boldsymbol{\theta}_{h}, \boldsymbol{\alpha}_{h}, p_{h}\right)\right\|_{-s-1, \widehat{G}_{1}} \\
& +C h^{\rho}\left(\left\|\boldsymbol{\theta}_{h}\right\|_{1, \widehat{G}_{1}}+\lambda^{-1 / 2} t\left\|\boldsymbol{\alpha}_{h}\right\|_{0, \widehat{G}_{1}}\right)+C\left\|\operatorname{grad} \psi_{h}\right\|_{0, \widehat{G}_{1}} .
\end{aligned}
$$

Proof. Suppose that $\widehat{G} \subset \subset \widehat{G}^{\prime} \subset \subset \widehat{G}_{1}$ are concentric spheres and that $\omega \equiv 1$ on $\widehat{G}$ and $\omega \in \mathbf{C}_{0}^{\infty}\left(\widehat{G}^{\prime}\right)$. Then we can write

$$
\left\|\boldsymbol{\theta}_{h}\right\|_{-s, \widehat{G}} \leq\left\|\omega \boldsymbol{\theta}_{h}\right\|_{-s, \widehat{G}_{1}}=\sup _{\mathbf{f} \in\left[\dot{H}^{s}\left(\widehat{G}_{1}\right)\right]^{2}} \frac{\left(\omega \boldsymbol{\theta}_{h}, \mathbf{f}\right)}{\|\mathbf{f}\|_{s}, \widehat{G}_{1}} .
$$

We consider the solution of (5.1)-(5.3) with $G=\widehat{G}_{1}$ and $g=\phi=0$ and we work as in the proof of Lemma 7.2. Then we apply Remark 4.1 to estimate the approximation errors and Lemma 5.3 to bound the solution of problem (5.1)-(5.3) in terms of $\|\mathbf{f}\|_{s, \widehat{G}_{1}}$.

Lemma 7.6. Let $\widehat{G}, \widehat{G}_{1}, s$ and $\rho$ be as in Lemma 7.5; then

$$
\begin{aligned}
\left\|p_{h}\right\|_{-s-1, \widehat{G}} \leq & C\left\|\left(\boldsymbol{\theta}_{h}, \boldsymbol{\alpha}_{h}, p_{h}\right)\right\|_{-s-1, \widehat{G}_{1}} \\
& +C h^{\rho}\left(\left\|\boldsymbol{\theta}_{h}\right\|_{1, \widehat{G}_{1}}+\lambda^{-1 / 2} t\left\|\boldsymbol{\alpha}_{h}\right\|_{0, \widehat{G}_{1}}\right)+C\left\|\operatorname{grad} \psi_{h}\right\|_{0, \widehat{G}_{1}} .
\end{aligned}
$$


Proof. Let $\widehat{G}, \widehat{G}^{\prime}, \widehat{G}_{1}$ and $\omega$ be as in the proof of the previous lemma. Then we have

$$
\left\|p_{h}\right\|_{-s-1, \widehat{G}} \leq\left\|\omega p_{h}\right\|_{-s-1, \widehat{G}_{1}}=\sup _{\substack{\stackrel{\circ}{f} \dot{\mathbf{H}}^{s+1}(\widehat{G})}} \frac{\left(\omega p_{h}, f\right)}{\|f\|_{s+1, \widehat{G}_{1}}} .
$$

We consider now the auxiliary problem (5.1)-(5.3) with $G=\widehat{G}_{1}, \mathbf{f}=\boldsymbol{\phi}=0$, and $g=f-\gamma \phi$, where $\gamma=\int_{\widehat{G}} f$ and $\phi \in \mathbf{C}_{0}^{\infty}\left(\widehat{G}_{1}\right)$ is such that $\int_{\widehat{G}_{1}} \phi=1$. Then with the same duality argument as before, we obtain (7.21).

Lemma 7.7. Let $\widehat{G}, \widehat{G}_{1}, s$, and $\rho$ be as in Lemma 7.5; then we have

$$
\begin{aligned}
\lambda^{-1 / 2} t\left\|\boldsymbol{\alpha}_{h}\right\|_{-s-1, \widehat{G}} \leq & C\left\|\left(\boldsymbol{\theta}_{h}, \boldsymbol{\alpha}_{h}, p_{h}\right)\right\|_{-s-1, \widehat{G}_{1}} \\
& +C h^{\rho}\left(\left\|\boldsymbol{\theta}_{h}\right\|_{1, \widehat{G}_{1}}+\lambda^{-1 / 2} t\left\|\boldsymbol{\alpha}_{h}\right\|_{0, \widehat{G}_{1}}\right) \\
& +C\left\|\operatorname{grad} \psi_{h}\right\|_{0, \widehat{G}_{1}} .
\end{aligned}
$$

Proof. The proof is similar to the preceding ones. Let $\widehat{G}, \widehat{G}^{\prime}, \widehat{G}_{1}$, and $\omega$ be as before. Then we have

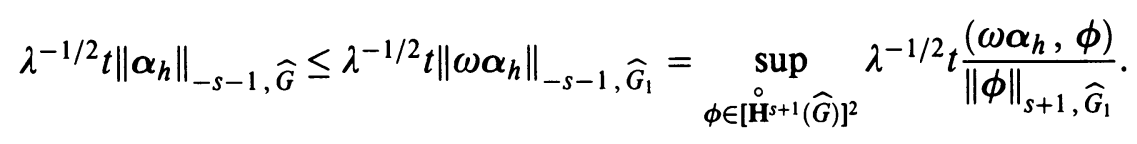

We consider the auxiliary problem (5.5)-(5.8) with $G=\widehat{G}_{1}$ and $\mathbf{f}=g=0$ and proceed as before, but using Lemma 5.5 instead of Lemma 5.3.

We collect the results of Lemmas 7.5-7.7 in the next proposition.

Proposition 7.8. Let $\widehat{G}$ and $\widehat{G}_{1}$ be concentric spheres with $\widehat{G}_{1} \subset \subset \Omega_{1}$, and let $s \geq 0$ be an integer; then for $\rho=\min (s+1, k)$, we have

$$
\begin{aligned}
\left\|\left(\boldsymbol{\theta}_{h}, \boldsymbol{\alpha}_{h}, p_{h}\right)\right\|_{-s, \widehat{G}} \leq & C\left\|\operatorname{grad} \psi_{h}\right\|_{0, \widehat{G}_{1}} \\
& +C\left(h^{\rho}\left\|\left(\boldsymbol{\theta}_{h}, \boldsymbol{\alpha}_{h}, p_{h}\right)\right\|_{1, \widehat{G}_{1}}+\left\|\left(\boldsymbol{\theta}_{h}, \boldsymbol{\alpha}_{h}, p_{h}\right)\right\|_{-s-1, \widehat{G}_{1}}\right) .
\end{aligned}
$$

We are now in a position to prove the following proposition, which gives Proposition 6.4 when we substitute $\boldsymbol{\theta}_{h}, \boldsymbol{\alpha}_{h}, p_{h}$, and $\psi_{h}$ by $\tilde{\boldsymbol{\theta}}_{h}-\boldsymbol{\theta}_{h}, \widetilde{\boldsymbol{\alpha}_{h}}-$ $\boldsymbol{\alpha}_{h}, \widetilde{p_{h}}-p_{h}$, and $P_{1}(\omega \psi)-\psi_{h}$, respectively.

Proposition 7.9. Let $G_{0}, G_{0}^{\prime}$ be concentric spheres; then for $h$ small enough and for $\mu \geq 0$ an integer, the following estimate holds:

$$
\left\|\left(\boldsymbol{\theta}_{h}, \boldsymbol{\alpha}_{h}, p_{h}\right)\right\|_{1, G_{0}} \leq C\left\|\left(\boldsymbol{\theta}_{h}, \boldsymbol{\alpha}_{h}, p_{h}\right)\right\|_{-\mu, G_{0}^{\prime}}+C\left\|\psi_{h}\right\|_{1, G_{0}^{\prime}} .
$$

Proof. Let $G_{1} \subset \subset G_{2} \subset \subset \cdots \subset \subset G_{\mu} \subset \subset G_{1}^{\prime}$ be concentric spheres; then we have from Proposition 7.8 with $\rho=1$ and $s=0$

$$
\begin{aligned}
\left\|\left(\boldsymbol{\theta}_{h}, \boldsymbol{\alpha}_{h}, p_{h}\right)\right\|_{0, G_{1}} \leq & C\left(h\left\|\left(\boldsymbol{\theta}_{h}, \boldsymbol{\alpha}_{h}, p_{h}\right)\right\|_{1, G_{2}}+\left\|\left(\boldsymbol{\theta}_{h}, \boldsymbol{\alpha}_{h}, p_{h}\right)\right\|_{-1, G_{2}}\right) \\
& +C\left\|\operatorname{grad} \psi_{h}\right\|_{0, G_{2}} .
\end{aligned}
$$

Iterating this argument for $s=-1, \ldots,-\mu+1$, we get

$$
\begin{aligned}
\left\|\left(\boldsymbol{\theta}_{h}, \boldsymbol{\alpha}_{h}, p_{h}\right)\right\|_{0, G_{1}} \leq & C\left(h\left\|\left(\boldsymbol{\theta}_{h}, \boldsymbol{\alpha}_{h}, p_{h}\right)\right\|_{1, G_{1}^{\prime}}+\left\|\left(\boldsymbol{\theta}_{h}, \boldsymbol{\alpha}_{h}, p_{h}\right)\right\|_{-\mu, G_{1}^{\prime}}\right) \\
& +C\left\|\operatorname{grad} \psi_{h}\right\|_{0, G_{1}^{\prime}} .
\end{aligned}
$$


Then we insert this inequality in (7.18) and obtain

$$
\begin{aligned}
\left\|\left(\boldsymbol{\theta}_{h}, \boldsymbol{\alpha}_{h}, p_{h}\right)\right\|_{1, G_{0} \leq} & C\left(h\left\|\left(\boldsymbol{\theta}_{h}, \boldsymbol{\alpha}_{h}, p_{h}\right)\right\|_{1, G_{1}^{\prime}}+\left\|\left(\boldsymbol{\theta}_{h}, \boldsymbol{\alpha}_{h}, p_{h}\right)\right\|_{-\mu, G_{1}^{\prime}}\right) \\
& +C\left\|\operatorname{grad} \psi_{h}\right\|_{0, G_{1}^{\prime}}
\end{aligned}
$$

Consider now a new sequence of concentric spheres $G_{1}^{\prime} \subset \subset G_{2}^{\prime} \subset \subset \cdots \subset \subset$ $G_{\mu+2}^{\prime}=G_{0}^{\prime}$; applying (7.25) with $G_{0}$ and $G_{1}^{\prime}$ replaced by $G_{j}^{\prime}$ and $G_{j+1}^{\prime}$, we obtain

$$
\begin{aligned}
\left\|\left(\boldsymbol{\theta}_{h}, \boldsymbol{\alpha}_{h}, p_{h}\right)\right\|_{1, G_{j}^{\prime} \leq} & C\left(h\left\|\left(\boldsymbol{\theta}_{h}, \boldsymbol{\alpha}_{h}, p_{h}\right)\right\|_{1, G_{j+1}^{\prime}}+\left\|\left(\boldsymbol{\theta}_{h}, \boldsymbol{\alpha}_{h}, p_{h}\right)\right\|_{-\mu, G_{j+1}^{\prime}}\right) \\
& +C\left\|\operatorname{grad} \psi_{h}\right\|_{0, G_{j+1}^{\prime}} .
\end{aligned}
$$

Starting with $G_{0}$ and iterating for $j=1, \ldots, \mu$, we get

$$
\begin{aligned}
\left\|\left(\boldsymbol{\theta}_{h}, \boldsymbol{\alpha}_{h}, p_{h}\right)\right\|_{1, G_{0} \leq} & C\left(h^{\mu+1}\left\|\left(\boldsymbol{\theta}_{h}, \boldsymbol{\alpha}_{h}, p_{h}\right)\right\|_{1, G_{\mu+1}^{\prime}}+\left\|\left(\boldsymbol{\theta}_{h}, \boldsymbol{\alpha}_{h}, p_{h}\right)\right\|_{-\mu, G_{\mu+1}^{\prime}}\right) \\
& +C\left\|\operatorname{grad} \psi_{h}\right\|_{0, G_{\mu+1}^{\prime}} .
\end{aligned}
$$

Now, let $G_{h}$ be a union of elements of $\mathscr{T}_{h}$ such that $G_{\mu+1}^{\prime} \subset \subset G_{h} \subset \subset G_{\mu+2}^{\prime}$; then by the inverse inequality we arrive at

$$
\begin{aligned}
h^{\mu+1}\left\|\left(\boldsymbol{\theta}_{h}, \boldsymbol{\alpha}_{h}, p_{h}\right)\right\|_{1, G_{\mu+1}^{\prime}} & \leq h^{\mu+1}\left\|\left(\boldsymbol{\theta}_{h}, \boldsymbol{\alpha}_{h}, p_{h}\right)\right\|_{1, G_{h}} \\
& \leq C\left\|\left(\boldsymbol{\theta}_{h}, \boldsymbol{\alpha}_{h}, p_{h}\right)\right\|_{-\mu, G_{h}} \leq C\left\|\left(\boldsymbol{\theta}_{h}, \boldsymbol{\alpha}_{h}, p_{h}\right)\right\|_{-\mu, G_{0}^{\prime}}
\end{aligned}
$$

The last two inequalities give (7.24).

Remark 7.10. The only point in this section where A3 has been used is the estimate of $T_{2}$ in the proof of Lemma 7.1. If A3 is not true (for example, this happens when rectangular families (3.43)-(3.46) are considered), we assume alternatively that $t \leq C h$ at the interior of $\Omega$. Then we can proceed as follows in order to get the bound of $T_{2}$ : in view of (3.19) we write

$$
\begin{aligned}
T_{2}= & \left(p_{h},-\operatorname{rot} \omega \cdot \mathbf{R}\left(\omega \boldsymbol{\alpha}_{h}\right)\right)+\left(P_{0}\left(\omega P_{0}\left(\omega p_{h}\right)\right), \operatorname{rot} \boldsymbol{\alpha}_{h}\right) \\
& +\left(P_{0}\left(\omega p_{h}\right)-\omega p_{h},-\operatorname{rot} \omega \cdot \boldsymbol{\alpha}_{h}\right)+\left(\omega p_{h},-\operatorname{rot} \omega \cdot \boldsymbol{\alpha}_{h}\right) .
\end{aligned}
$$

Then, since $t \leq C h$, we obtain

$$
\begin{aligned}
\lambda^{-1} t^{2} T_{2} \leq & C h^{2}\left\|p_{h}\right\|_{0, G_{1}^{\prime}}+C h^{2} \lambda^{-1} t^{2}\left\|\boldsymbol{\alpha}_{h}\right\|_{0, G_{1}^{\prime}}+\left(P_{0}\left(\omega P_{0}\left(\omega p_{h}\right)\right), \operatorname{rot} \boldsymbol{\alpha}_{h}\right) \\
& +\left(p_{h},-\operatorname{rot} \omega \cdot \mathbf{R}\left(\omega \boldsymbol{\alpha}_{h}\right)\right)+\left(\omega p_{h},-\operatorname{rot} \omega \cdot \boldsymbol{\alpha}_{h}\right),
\end{aligned}
$$

and inserting this inequality in (7.7) instead of (7.11), we arrive at (7.6).

\section{INTERIOR ASYMPTOTIC RATES OF CONVERGENCE}

In this section we shall apply the abstract results of $\S 6$ to the families of finite element spaces introduced in $\S 4$ and state the asymptotic interior estimates. To this end, we need to evaluate the rate of convergence of the negative norms of the errors which appear in the second members of (6.1), (6.24), and (6.26). As in $\S 6$, we proceed by analyzing separately the two Poisson equations and the Stokes-like system.

It is easy to obtain from (6.1), by means of a duality argument with $\mu_{1}=k-1$ and $s=k+1$, the following bound:

$$
\left\|\psi-\psi_{h}\right\|_{1, \Omega_{0}} \leq C h^{k}\left(\|\psi\|_{k+1, \Omega_{1}}+\|\psi\|_{1, \Omega}\right) .
$$


More delicate is the estimate of $\left\|\left(\boldsymbol{\theta}-\boldsymbol{\theta}_{h}, \boldsymbol{\alpha}-\boldsymbol{\alpha}_{h}, p-p_{h}\right)\right\|_{-\mu_{2}, \Omega_{1}}$, because of the presence of the boundary layer in the solution of the system (3.34)-(3.36). We carry out the proof of the estimate of such a quantity by duality arguments.

First we prove the following lemma, which will be useful in the remaining part of the section.

Lemma 8.1. Consider the solution $(\mathbf{z}, r, \chi)$ of (5.5)-(5.8); then for $s+\sigma \leq k$ there holds

$$
\begin{aligned}
\left(\boldsymbol{\theta}-\boldsymbol{\theta}_{h}, \mathbf{f}\right)+\lambda^{-1} t^{2}\left(\boldsymbol{\alpha}-\boldsymbol{\alpha}_{h}, \boldsymbol{\phi}\right)+\left(p-p_{h}, g\right) \\
\leq C h^{s+\sigma}\left(\|\boldsymbol{\theta}\|_{s+1}+\|p\|_{s}+\lambda^{-1 / 2} t\|p\|_{s+1}+\lambda^{-1 / 2} t\|\boldsymbol{\alpha}\|_{s}+\|\psi\|_{s+\sigma+1}\right) \\
\quad \times\left(\|\mathbf{z}\|_{\sigma+1}+\|r\|_{\sigma}+\lambda^{-1 / 2} t\|r\|_{\sigma+1}+\lambda^{-1 / 2} t\|\boldsymbol{\chi}\|_{\sigma}\right) .
\end{aligned}
$$

Proof. We multiply (5.5) by $\boldsymbol{\theta}-\boldsymbol{\theta}_{h},(5.6)$ by $p-p_{h},(5.7)$ by $\boldsymbol{\alpha}-\boldsymbol{\alpha}_{h}$, and add the three equations. Then we use (6.11)-(6.13), integration by parts, (3.19) and obtain

$$
\begin{aligned}
&\left(\boldsymbol{\theta}-\boldsymbol{\theta}_{h}, \mathbf{f}\right)+\lambda^{-1} t^{2}\left(\boldsymbol{\alpha}-\boldsymbol{\alpha}_{h}, \boldsymbol{\phi}\right)+\left(p-p_{h}, g\right) \\
&= \mathbf{a}\left(\boldsymbol{\theta}-\boldsymbol{\theta}_{h}, \mathbf{z}-\Pi \mathbf{z}\right)-\left(\operatorname{rot}\left(\boldsymbol{\theta}-\boldsymbol{\theta}_{h}\right), r-P_{0} r\right)-\left(p-p_{h}, \operatorname{rot}(\mathbf{z}-\Pi \mathbf{z})\right) \\
&-\lambda^{-1} t^{2}\left(\operatorname{rot}(\boldsymbol{\chi}-\mathbf{R} \boldsymbol{\chi}), p-P_{0} p\right)+\lambda^{-1} t^{2}\left(\boldsymbol{\chi}-\mathbf{R} \boldsymbol{\alpha}, \boldsymbol{\alpha}-\boldsymbol{\alpha}_{h}\right) \\
&-\lambda^{-1} t^{2}\left(\operatorname{rot}\left(r-P_{0} r\right), \boldsymbol{\alpha}-\boldsymbol{\alpha}_{h}\right)+(\operatorname{grad} \psi, \Pi \mathbf{z}-\mathbf{R} \Pi \mathbf{z}) \\
&-\left(\operatorname{grad}\left(\psi-\psi_{h}\right), \mathbf{R} \Pi \mathbf{z}\right) .
\end{aligned}
$$

Applying the approximation properties of the finite element spaces and the error estimates (3.52), we get

$$
\begin{aligned}
\left(\boldsymbol{\theta}-\boldsymbol{\theta}_{h}, \mathbf{f}\right)+\lambda^{-1} t^{2}\left(\boldsymbol{\alpha}-\boldsymbol{\alpha}_{h}, \boldsymbol{\phi}\right)+\left(p-p_{h}, g\right) & \\
\leq & C h^{s+\sigma}\left(\|\boldsymbol{\theta}\|_{s+1}+\|p\|_{s}+\lambda^{-1 / 2} t\|p\|_{s+1}+\lambda^{-1 / 2} t\|\boldsymbol{\alpha}\|_{s}\right) \\
& \times\left(\|\mathbf{z}\|_{\sigma+1}+\|r\|_{\sigma}+\lambda^{-1 / 2} t\|r\|_{\sigma+1}+\lambda^{-1 / 2} t\|\boldsymbol{\chi}\|_{\sigma}\right) \\
& +(\operatorname{grad} \psi, \Pi \mathbf{z}-\mathbf{R} \Pi \mathbf{z})-\left(\operatorname{grad}\left(\psi-\psi_{h}\right), \mathbf{R} \Pi \mathbf{z}\right) .
\end{aligned}
$$

The last term can be bounded using the standard estimates for the approximation of the Poisson equation by conforming elements as follows:

$$
\left(\operatorname{grad}\left(\psi-\psi_{h}\right), \mathbf{R} \Pi \mathbf{z}\right) \leq C h^{s+\sigma}\|\psi\|_{s+\sigma+1}\|\mathbf{z}\|_{1},
$$

while for the next to the last term we can use the same argument already employed in the proof of Corollary $6.2(\operatorname{see}(6.21))$ : let $(\operatorname{grad} \psi)^{*}$ be the interpolant of $\operatorname{grad} \psi$ in $\mathscr{L}_{s-1}$; then

$$
(\operatorname{grad} \psi, \Pi \mathbf{z}-\mathbf{R} \Pi \mathbf{z}) \leq C h^{s+\sigma}\|\psi\|_{s}\|\mathbf{z}\|_{\sigma+1} .
$$

Then (8.2) is obtained.

Now recalling the definitions of the norms (2.1) and (2.2) and the a priori estimates for the dual problem (5.5)-(5.8), see Lemmas 5.1, 5.2, and 5.4, it is easy to obtain the following asymptotic rates of convergence.

Theorem 8.2. Let $k=2$ or 3 in (3.38)-(3.41) and (3.43)-(3.46); then for $F$ sufficiently smooth,

$$
\begin{aligned}
& \left\|\left(\boldsymbol{\theta}-\boldsymbol{\theta}_{h}, \boldsymbol{\alpha}-\boldsymbol{\alpha}_{h}, p-p_{h}\right)\right\|_{1, \Omega_{0}} \\
& \quad \leq C h^{k}\left(\|\boldsymbol{\theta}\|_{k+1, \Omega_{1}}+\|p\|_{k, \Omega_{1}}+\lambda^{-1 / 2} t\|p\|_{k+1, \Omega_{1}}+\|\psi\|_{k+1, \Omega_{1}}+C(\boldsymbol{F})\right) .
\end{aligned}
$$


Proof. It remains to evaluate $\left\|\left(\boldsymbol{\theta}-\boldsymbol{\theta}_{h}, \boldsymbol{\alpha}-\boldsymbol{\alpha}_{h}, p-p_{h}\right)\right\|_{-\mu_{2}, \Omega}$ (see Corollary 6.2 and Remark 6.6). Let us fix $k=2$. We choose $\mu_{2}=0$ and estimate separately the three terms entering the norm by means of a duality argument. We have

$$
\left\|\boldsymbol{\theta}-\boldsymbol{\theta}_{h}\right\|_{0}=\sup _{\mathbf{f} \in\left[\mathbf{L}^{2}(\Omega)\right]^{2}} \frac{\left(\boldsymbol{\theta}-\boldsymbol{\theta}_{h}, \mathbf{f}\right)}{\|\mathbf{f}\|_{0}} .
$$

Therefore, we consider the solution of (5.5)-(5.8) corresponding to $g=\phi=0$ and $\mathbf{f} \in\left[\mathbf{L}^{2}(\Omega)\right]^{2}$ and apply Lemma 8.1 with $s=\sigma=1$. By the global error estimate (3.11) and the a priori estimate (5.10) it follows that

$$
\left\|\boldsymbol{\theta}-\boldsymbol{\theta}_{h}\right\|_{0} \leq C h^{2}\|F\|_{0}
$$

Analogously, if we consider (5.5)-(5.8) with $\mathbf{f}=\phi=0$ and $g \in \widehat{\mathbf{H}}_{0}^{1}(\Omega)$ and apply Lemma $8.1,(3.11)$, and $(5.10)$, we get

$$
\left\|p-p_{h}\right\|_{-1}=\sup _{g \in \mathbf{H}_{0}^{1}(\Omega)} \frac{\left(p-p_{h}, g\right)}{\|g\|_{1}} \leq C h^{2}\|F\|_{0} .
$$

The next term we must bound is

$$
\lambda^{-1 / 2} t\left\|\boldsymbol{\alpha}-\boldsymbol{\alpha}_{h}\right\|_{-1}=\frac{1}{\lambda^{-1 / 2} t} \sup _{\mathbf{f} \in\left[\mathbf{L}^{2}(\Omega)\right]^{2}} \lambda^{-1} t^{2} \frac{\left(\boldsymbol{\alpha}-\boldsymbol{\alpha}_{h}, \phi\right)}{\|\phi\|_{0}} .
$$

Hence, in (5.5)-(5.8) we take $\mathbf{f}=g=0$ and $\phi \in\left[\mathbf{H}_{0}^{1}(\Omega)\right]^{2}$ and apply Lemma $8.1,(3.11)$, and Lemma 5.4 to arrive at

$$
\lambda^{-1 / 2} t\left\|\boldsymbol{\alpha}-\boldsymbol{\alpha}_{h}\right\|_{-1} \leq C h^{2}\|F\|_{0} .
$$

The estimates (8.6)-(8.8) inserted in Corollary 6.2 imply (8.5).

For $k=3$ the proof is similar. The only differences consist in choosing $\mu_{2}=2, s=\sigma=\frac{3}{2}$ in Lemma 8.1 and in applying (3.12) instead of (3.11), (5.11), and (5.14) instead of (5.10) and (5.13), so that

$$
\left\|\left(\boldsymbol{\theta}-\boldsymbol{\theta}_{h}, \boldsymbol{\alpha}-\boldsymbol{\alpha}_{h}, p-p_{h}\right)\right\|_{-2} \leq C(F) h^{3}
$$

with $C(F)$ depending on the $\mathbf{H}^{1}$-norm of $F$.

Finally, we have the following asymptotic error estimate for the displacement:

$$
\left\|w-w_{h}\right\|_{1, \Omega_{0}} \leq C h^{k}\left(\|\psi\|_{k+1, \Omega_{1}}+\|w\|_{k+1, \Omega_{1}}+\|(\boldsymbol{\theta}, \boldsymbol{\alpha}, p)\|_{k+1, \Omega_{1}}+C(F)\right),
$$

for $k=2,3$.

This bound can be obtained from (6.27) and (8.1), (8.6)-(8.9), using again a duality argument to estimate $\left\|w-w_{h}\right\|_{-\mu_{3}, \Omega}$. More precisely, we have

$$
\left\|w-w_{h}\right\|_{-\mu_{3}}=\sup _{f \in \mathbf{H}^{\mu_{3}(\Omega)}} \frac{\left(w-w_{h}, f\right)}{\|f\|_{\mu_{3}}} .
$$

Let $u$ be the solution of the Dirichlet problem $-\Delta u=f$ in $\Omega$ and $u=0$ on 
$\partial \Omega$; then the numerator in (8.11) becomes (see (6.25) and (6.21))

$$
\begin{aligned}
\left(w-w_{h}, f\right)= & \left(\operatorname{grad}\left(w-w_{h}\right), \operatorname{grad}\left(u-P_{1} u\right)\right) \\
& +\left(\boldsymbol{\theta}-\mathbf{R} \boldsymbol{\theta}_{h}, \operatorname{grad}\left(P_{1} u-u\right)\right)+\left(\boldsymbol{\theta}-\mathbf{R} \boldsymbol{\theta}_{h}, \operatorname{grad} u\right) \\
= & \left(\operatorname{grad}\left(w-w_{h}\right), \operatorname{grad}\left(u-P_{1} u\right)\right)+\left(\boldsymbol{\theta}-\mathbf{R} \boldsymbol{\theta}, \operatorname{grad}\left(P_{1} u-u\right)\right) \\
& +\left(\mathbf{R}\left(\boldsymbol{\theta}-\boldsymbol{\theta}_{h}\right), \operatorname{grad}\left(P_{1} u-u\right)\right)+\left(\boldsymbol{\theta}-\boldsymbol{\theta}_{h}, \operatorname{grad} u\right) \\
& +\left(\boldsymbol{\theta}_{h}-\boldsymbol{\theta}-\mathbf{R}\left(\boldsymbol{\theta}_{h}-\boldsymbol{\theta}\right), \operatorname{grad} u-(\operatorname{grad} u)^{*}\right) \\
& +\left(\boldsymbol{\theta}-\mathbf{R} \boldsymbol{\theta}, \operatorname{grad} u-(\operatorname{grad} u)^{*}\right),
\end{aligned}
$$

where $(\operatorname{grad} u)^{*}$ is the interpolant of $\operatorname{grad} u$ in $\mathscr{L}_{k-2}$. Then, using appropriately the approximation properties of the finite element spaces together with (8.1), (8.6)-(8.9), the inequality (8.10) follows for suitable choices of $\mu_{3}$ in the two cases $k=2$ and $k=3$.

This method of proof cannot be extended to obtain the estimates (8.5) and (8.10) for all the values of $k$ greater than 3 . In fact, in order to use the duality argument as before, we should have an a priori estimate for the solution $(\mathbf{z}, r)$ of problem (5.1)-(5.3), with $g=\phi=0$ and $\mathbf{f} \in\left[\mathbf{H}_{0}^{\mu_{2}}\right]^{2}$, of the form $\|\mathbf{z}\|_{\sigma+1} \leq$ $C\|\mathbf{f}\|_{\mu_{2}}$ with $\sigma>3 / 2$. We show that this is impossible.

Since $\operatorname{rot}\left(\mathbf{z}+\lambda^{-1} t^{2} \operatorname{rot} r\right)=0$, we have for some element $\eta$ of $\mathbf{H}_{0}^{1}$ that

$$
\lambda^{-1 / 2} t^{2} \operatorname{rot} r=\operatorname{grad} \eta-\mathbf{z}
$$

Substituting this in (5.1), we obtain the following equivalent formulation:

$$
\begin{gathered}
\mathbf{A z}=\lambda t^{-2}(\operatorname{grad} \eta-\mathbf{z})+\mathbf{f} \quad \text { in } \Omega, \\
\operatorname{div}(\operatorname{grad} \eta-\mathbf{z})=0 \quad \text { in } \Omega, \\
\mathbf{z}=0, \quad \eta=0, \quad \text { on } \partial \Omega .
\end{gathered}
$$

Following Arnold and Falk [2], we obtain that $\mathrm{z}$ and $\eta$ can be well approximated by the following expansions:

$$
\begin{gathered}
\eta=\eta_{0}+\lambda^{-1} t^{2} \eta_{2}+\lambda^{-3 / 2} t^{3} \eta_{3}+\cdots, \\
\mathbf{z}=\mathbf{z}_{0}+\lambda^{-1} t^{2} \mathbf{z}_{2}+\lambda^{-3 / 2} t^{3} \mathbf{z}_{3}+\cdots+\chi\left(\lambda^{-3 / 2} t^{3} \operatorname{rot} Q_{3}\left(\rho / \lambda^{-1 / 2} t, \tau\right)+\cdots\right),
\end{gathered}
$$

where $\rho$ and $\tau$ denote the boundary-fitted coordinates and $\chi$ is a cutoff function (see [2]). The interior terms in the expansions can be constructed as follows, setting $C=E / 12\left(1-\nu^{2}\right)$ :

$$
\begin{gathered}
C \Delta^{2} \eta_{i}= \begin{cases}-\operatorname{div} \mathbf{f}, & i=0, \\
0, & i>0,\end{cases} \\
\mathbf{z}_{i}= \begin{cases}\operatorname{grad} \eta_{i}, & i=0,1, \\
\operatorname{grad}\left(\eta_{2}+C \Delta \eta_{0}\right)+\mathbf{f}, & i=2, \\
\operatorname{grad}\left(\eta_{i}+C \Delta \eta_{i-2}\right)+\cdots, & i>2,\end{cases}
\end{gathered}
$$

while for the boundary terms we have (set $\left.\hat{\rho}=\rho / \lambda^{-1 / 2} t\right)$

$$
-\frac{\partial^{2} Q_{i}}{\partial \hat{\rho}^{2}}+Q_{i}=\cdots,
$$

where in the right-hand side appear the preceding terms of the expansion. To these equations suitable boundary conditions have to be added according to 
(8.14). These boundary conditions are such that the boundary terms $Q_{i}$ have an exponential decay as $\rho$ tends to infinity, that is, $Q_{i}=q_{i}(\tau) e^{-\rho / \lambda^{-1 / 2} t}$. Recalling that $\left\|e^{-\rho / \lambda^{-1 / 2} t}\right\|_{0} \leq C t^{1 / 2}$, we see that $\|\mathbf{z}\|_{\sigma+1}$ is bounded uniformly for $\sigma>$ $3 / 2$ only if $Q_{3}=0$. But $Q_{3}$ solves

$$
\begin{array}{cc}
-\frac{\partial^{2} Q_{3}}{\partial \hat{\rho}^{2}}+Q_{3}=0, & \hat{\rho}>0, \\
\frac{\partial Q_{3}}{\partial \hat{\rho}}=\frac{\partial}{\partial \tau} \Delta \eta_{0}, & \hat{\rho}=0 ;
\end{array}
$$

hence, we must find out for which $\mathbf{f} \in\left[\mathrm{H}_{0}^{\mu_{2}}(\Omega)\right]^{2}$ it is true that the solution of

$$
\begin{cases}C \Delta^{2} \eta_{0}=-\operatorname{div} \mathbf{f}, & \text { in } \Omega, \\ \eta_{0}=0, & \text { on } \partial \Omega, \\ \frac{\partial \eta_{0}}{\partial n}=0, & \text { on } \partial \Omega,\end{cases}
$$

satisfies the additional condition $\frac{\partial}{\partial \tau} \Delta \eta_{0}=0$.

This is equivalent to describing the set whose elements are $\Delta^{2} v$, when $v$ belongs to

$$
V=\left\{v \in \mathbf{H}^{3}(\Omega): v=0, \frac{\partial v}{\partial n}=0, \frac{\partial}{\partial \tau} \Delta v=0 \text { on } \partial \Omega\right\},
$$

which can be characterized as

$$
V=\mathbf{H}_{0}^{3}(\Omega) \oplus \operatorname{span}\left(\hat{\rho}^{2} \chi\right) .
$$

Now we observe that if $v \in \mathbf{H}_{0}^{3}(\Omega)$, then $\Delta^{2} v$ is orthogonal to all functions $\phi$ such that $\Delta^{2} \phi=0$ in $\Omega$ and $\phi=0$ and $\partial \Omega$. Therefore, the set $\left\{\Delta^{2} v \mid v \in V\right\}$ is not dense in any $\mathbf{H}_{0}^{s}$-space. This proves that for arbitrary $\mathbf{f}$ vanishing on $\partial \Omega, Q_{3}$ is different from zero, so that $\|\mathbf{z}\|_{\sigma+1}=O\left(t^{-\sigma+3 / 2}\right)$.

\section{BIBLIOGRAPHY}

1. D. N. Arnold and R. S. Falk, The boundary layer for the Reissner-Mindlin plate model, SIAM J. Math. Anal. 26 (1989), 1276-1290.

2. __ Edge effects in the Reissner-Mindlin plate theory, Analytic and Computational Models of Shells, A.S.M.E., New York, 1989.

3. K. J. Bathe, Finite element procedures in engineering analysis, Prentice-Hall, Englewood Cliffs, NJ, 1982.

4. J. H. Bramble, J. A. Nitsche, and A. H. Schatz, Maximum-norm interior estimates for Ritz-Galerkin methods, Math. Comp. 29 (1975), 677-688.

5. F. Brezzi, On the existence, uniqueness and approximations of saddle point problems arising from Lagrangian multipliers, RAIRO Anal. Numér. 2 (1974), 129-151.

6. F. Brezzi, K. J. Bathe, and M. Fortin, Mixed-interpolated elements for Reissner-Mindlin plates, Internat. J. Numer. Methods Eng. 28 (1989), 1787-1801.

7. F. Brezzi and M. Fortin, Numerical approximation of Mindlin-Reissner plates, Math. Comp. 47 (1986), 151-158.

8. _ـ Mixed and hybrid finite element methods, Springer-Verlag, New York, 1991.

9. F. Brezzi, M. Fortin, and R. Stenberg, A complete error analysis of some Reissner-Mindlin plate bending elements, Math. Models Methods Appl. Sci. 1 (1991), 125-151.

10. T. Dupont and R. Scott, Polynomial approximation of functions in Sobolev spaces, Math. Comp. 34 (1990), 441-463. 
11. L. Gastaldi, Uniform interior error estimates for Reissner-Mindlin plate model, Pubbl. IAN/CNR, N. 802, Pavia, 1991.

12. L. Gastaldi and R. H. Nochetto, Quasi-optimal pointwise error estimates for the ReissnerMindlin plate, SIAM J. Numer. Anal. 28 (1991), 363-377.

13. J. Necăs, Les méthodes directes en théorie des équations elliptiques, Masson, Paris, 1967.

14. J. A. Nitsche and A. H. Schatz, Interior estimates for Ritz-Galerkin methods, Math. Comp. 28 (1974), 937-958.

15. R. Scholtz, Optimal $\mathbf{L}^{\infty}$-estimates for a mixed finite element method for second order elliptic and parabolic problems, Calcolo 20 (1983), 355-377.

16. L. R. Scott and S. Zhang, Finite element interpolation of nonsmooth functions satisfying boundary conditions, Math. Comp. 54 (1990), 483-493.

Dipartimento di Matematica, Universita di Pavia, 27100 Pavia, Italy

E-mail address: gastaldil@ipv85.unipv.it 